\title{
Kinematics and dynamics of the luminous infrared galaxy pair NGC 5257/58 (Arp 240)^
}

\author{
I. Fuentes-Carrera ${ }^{1}$, M. Rosado ${ }^{2}$, P. Amram ${ }^{3}$, E. Laurikainen ${ }^{4}$, H. Salo ${ }^{4}$, J. A. Gómez-López ${ }^{3}$, H. O. Castañeda ${ }^{1}$ \\ A. Bernal ${ }^{2}$, and C. Balkowski ${ }^{5}$ \\ ${ }^{1}$ Escuela Superior de Física y Matemáticas, Instituto Politécnico Nacional (ESFM-IPN), U.P. Adolfo López Mateos, edificio 9 , \\ Zacatenco, 07730 Mexico City, Mexico \\ e-mail: isaura@esfm.ipn.mx, isaura.fuentescarrera@gmail.com \\ 2 Instituto de Astronomía, Universidad Nacional Autónoma de México (UNAM), Apdo. Postal 70-264, 04510 Mexico City, Mexico \\ 3 Aix-Marseille Université, CNRS, LAM (Laboratoire d'Astrophysique de Marseille), 13388 Marseille, France \\ ${ }^{4}$ Department of Physical Sciences, Division of Astronomy, University of Oulu, 90570 Oulu, Finland \\ 5 GEPI, Observatoire de Paris, CNRS, Université Paris Diderot, 5 Place Jules Janssen, 92190 Meudon, France
}

Received 29 August 2018 / Accepted 19 October 2018

\begin{abstract}
Context. Encounters between galaxies modify their morphology, kinematics, and star formation history. The relation between these changes and external perturbations is not straightforward. The great number of parameters involved requires both the study of large samples and individual encounters where particular features, motions, and perturbations can be traced and analysed in detail.

Aims. We analysed the morphology, kinematics, and dynamics of two luminous infrared spiral galaxies of almost equal mass, NGC 5257 and NGC 5258, in which star formation is mostly confined to the spiral arms, in order to understand interactions between galaxies of equivalent masses and star-forming processes during the encounter.

Methods. Using scanning Fabry-Perot interferometry, we studied the contribution of circular and non-circular motions and the response of the ionized gas to external perturbations. We compared the kinematics with direct images and traced the star-forming processes and gravitational effects due to the presence of the other galaxy. The spectral energy distribution of each member of the pair was fitted. A mass model was fitted to the rotation curve of each galaxy.

Results. Large, non-circular motions detected in both galaxies are associated with a bar, spiral arms, and HII regions for the inner parts of the galaxies, and with the tidal interaction for the outer parts of the discs. Bifurcations in the rotation curves indicate that the galaxies have recently undergone pericentric passage. The pattern speed of a perturbation of one of the galaxies is computed. Location of a possible corotation seems to indicate that the gravitational response of the ionized gas in the outer parts of the disc is related to the regions where ongoing star formation is confined. The spectral energy distribution fit indicates slightly different star formation history for each member of the pair. For both galaxies, a pseudo-isothermal halo better fits the global mass distribution.
\end{abstract}

Key words. galaxies: interactions - galaxies: kinematics and dynamics - galaxies: spiral - galaxies: star formation galaxies: individual: NGC 5257 - galaxies: individual: NGC 5258

\section{Introduction}

Observations of distant galaxies suggest that mergers of galaxies played important roles in the formation of stars and galaxies in early epochs (e.g. Schreiber et al. 2015; Rodrigues et al. 2017). Studying the interacting galaxy population in the local universe is an important step towards a better understanding of the process of galaxy formation and evolution. The way the interaction process triggers, sustains, or inhibits the formation of structure in spiral galaxies has been studied, from broad statistical studies to more detailed morphological and photometric analysis (e.g. Delgado-Serrano et al. 2010; Martinez-Valpuesta et al. 2017). The kinematics and dynamics of a galaxy are also affected by encounters and interactions. Tidal features in interacting galaxies can give information on the stage of the encounter through kinematical effects on the baryonic matter, as well

\footnotetext{
* The reduced data cube is only available at the CDS via anonymous ftp to cdsarc.u-strasbg.fr $(130.79 .128 .5)$ or via http: //cdsarc.u-strasbg.fr/viz-bin/qcat?J/A+A/621/A25
}

as the difference in rotation curves according to environment (e.g. Pedrosa et al. 2008). Several attempts have been made to kinematically constraint the evolutionary scenario of interactions (e.g. Ji et al. 2014; Bellocchi et al. 2016) and the formation of structures such as bars and tidal arms, amongst others (e.g. Fuentes-Carrera et al. 2010; Zaragoza-Cardiel et al. 2015). Early numerical simulations of interacting galaxies sought to reproduce the observed morphology; however, it has been shown that kinematical information imposes important restrictions on any interaction model (e.g. Salo \& Laurikainen 1993, 2000a,b; Gabbasov et al. 2009, 2014).

The correlation between enhanced star formation (SF) and external perturbations is not straightforward (e.g. Scudder et al. 2015; Davies et al. 2015; Krabbe et al. 2017). In most studies using the partly or completely far-infrared (FIR) flux to estimate star formation rates (SFR) in galaxies, a clear enhancement is found in interacting systems (e.g. Ellison et al. 2013). In 1999, Gao and Solomon showed that the star formation efficiency (SFE) increases as separation between merger nuclei decreases, suggesting that the starburst phase occurs at a 
late stage of merging and is confined to the central kiloparsec (Gao \& Solomon 1999). This effect has been shown for example in the work of Yamashita et al. (2017). Optical, nearinfrared (NIR), mid-infrared (MIR), and radio continuum studies also imply enhanced central SF in interacting galaxies (e.g. Smith et al. 2007; Patton et al. 2011; Ellison et al. 2013; Brassington et al. 2015). However, when unbiased samples of interacting and non-interacting galaxies are compared, the optical studies show only a moderate excess of SF concentrated in the centres of the interacting galaxies (e.g. Bergvall et al. 2003; Knapen et al. 2015).

Regarding the role of dark matter (DM) in the dynamics and evolution of interacting galaxies, the picture is less clear. In the standard picture of hierarchical structure formation, larger DM halos are built up through the accretion and merger of smaller halos. Most of the work on the dependence of DM halos on environment has been done using statistical results from large numerical simulations (e.g. Haas et al. 2012). Observationally, the structure and distribution of DM halos has been found to be related to the environment of their galaxies (e.g. Hudson et al. 2015). Following the hierarchical scenario and observations, isolated galaxies at a given cosmic epoch have formed pairs, triplets, and groups at later stages of evolution; some of those structures join to form galaxy clusters. From field galaxies to galaxies in rich clusters, galaxies should merge together to eventually form larger structures; dark halos being much more extended than their embedding galaxies, at some point the mass in the galaxies' individual halos must migrate into a shared halo. Numerical simulations indicate that this process starts when the distance between galaxies reaches the halo virial radius (Kazantzidis et al. 2004; Springel et al. 2008). However, this is not directly observed and the details of the redistribution of DM during these encounters is poorly understood. Galaxy interactions are still the best option to trace and probe the matter redistribution during such events. Those processes being dynamical, the morphological information on the encounter, even if instructive in the case of for example tidal tails and plume formation, is not sufficient to characterise the interaction. Kinematical data are needed to understand the dynamics of the system and its three-dimensional (3D) geometry.

We present and analyse scanning Fabry-Perot (FP) interferometric observations of the interacting galaxy pair Arp 240 (KPG 389), composed of two luminous infrared galaxies (LIRGs), NGC 5257 and NGC 5258. Section 2 presents the observations and data reduction. Section 3 introduces the pair of galaxies. In Sect. 4 we discuss the kinematic information derived from the observations along with direct images of the pair in different wavelengths in order to compare between kinematical and morphological features. Section 5 presents dynamical results, mass estimates, and mass distribution. In Sect. 6 we show the fit of the spectral energy distribution (SED) of both galaxies with estimates of their star formation history. A discussion is presented in Sect. 7 and conclusions are presented in Sect. 8. The cosmological model adopted in this work has $H_{0}=67.8 \mathrm{~km} \mathrm{~s}^{-1} \mathrm{Mpc}^{-1}$, $\Omega_{\mathrm{m}}=0.308$, and $\Omega_{\lambda}=0.692$ (Planck Collaboration XIII 2016).

\section{Observations and data reductions}

Observations of Arp 240 were done at the $2.1 \mathrm{~m}$ telescope of the Observatorio Astronómico Nacional in San Pedro Mártir, Mexico (OAN-SPM) using the scanning Fabry-Perot interferometer PUMA (Rosado et al. 1995). PUMA is a focal reducer used to make direct imagery and Fabry-Perot (FP) interferometry of extended emission sources. Instrumental specifications
Table 1. Instrumental and observational parameters.

\begin{tabular}{ll}
\hline \hline Parameter & Value \\
\hline Telescope & $2.1 \mathrm{~m}(\mathrm{OAN}-\mathrm{SPM})$ \\
Instrument & PUMA \\
Scanning F-P interferometer & ET-50 (Queensgate) \\
Interference order & 330 at $6562.78 \AA$ \\
Free spectral range, FSR $\left(\AA / \mathrm{km} \mathrm{s}^{-1}\right)$ & $19.95 / 912.0$ \\
Interferometer finesse, $\mathcal{F}$ & 24 \\
Number of scanning channels & 48 \\
Spectral sampling at H $\alpha\left(\AA / \mathrm{km} \mathrm{s}^{-1}\right)$ & $0.42 / 19.0$ \\
Spectral resolution, $R$ & $6720 / 0.83$ \\
Spectral element $\left(\AA / \mathrm{km} \mathrm{s}{ }^{-1}\right)$ & $0.83 / 38.5$ \\
Interference filter $(\AA)$ & 6720 \\
$F W H M$ of filter $(\AA)$ & 20 \\
Detector & Tektronix CCD \\
Detector size (px $\times$ px) & $1024 \times 1024$ \\
Image scale (" per px) & 0.58 \\
Electronic binning & 2 \\
Image scale after binning $\left({ }^{\prime \prime}\right.$ per px) & 1.16 \\
Mean seeing during observations (") & 1.5 \\
Total exposure time $(\mathrm{min})$ & 96 \\
Total exposure time per cube (min) & 48 \\
Number of science cubes & 2 \\
Exposure time per channel $(\mathrm{s})$ & 60 \\
Exposure time for direct image (s) & 120 \\
Calibration line $(\AA)$ & $6717(\mathrm{Ne}$ lamp) \\
\hline
\end{tabular}

are presented in Table 1 . A $1024 \times 1024$ Tektronix CCD detector was used with a sampling of $0.58^{\prime \prime} /$ px per axis. A physical $2 \times 2$ binning in both spatial dimensions was done by the CCD controller in order to enhance the signal-to-noise ratio $(\mathrm{S} / \mathrm{N})$ in the readout noise per pixel by a factor of four. The final spatial sampling thus equals to $1.16^{\prime \prime} / \mathrm{px}$. In order to isolate the redshifted $\mathrm{H} \alpha$ emission of the galaxies, an interference filter centred at $6720 \AA$ with a full width at half maximum (FWHM) of $20 \AA$ was used. The interference filter having a Gaussian-like shape allows us to pass wavelengths ranging from $6706 \AA$ to $6734 \AA$. To average the sky variations during the exposure, we got two data cubes with an exposure time of $48 \mathrm{~min}$ each $(60 \mathrm{~s}$ per channel times 48 channels), which were co-added leading to a total exposure time of $96 \mathrm{~min}$. For the calibration we used a Ne lamp since the $6717 \AA \mathrm{Ne}$ line was close to the redshifted $\mathrm{H} \alpha$ galaxy wavelength. Two calibration cubes were obtained at the beginning and at the end of the exposure in order to account for metrology. Observational parameters are shown in Table 1.

Data reduction and analysis were done using mainly the ADHOCw software developed by J. Boulesteix (Amram et al. 1989) and the CIGALE software (Le Coarer et al. 1993). Standard corrections (cosmic rays removal, bias subtraction, and flatfielding) were done on each data cube before co-addition. Once co-added, using the calibration data cube, the resulting science data cube was calibrated in wavelength, meaning that each profile for each pixel has the same wavelength origin (and range). Then the night sky continuum and $\mathrm{OH}$ night sky lines were subtracted. A spectral Gaussian smoothing (with a velocity dispersion equal to two channels) has been applied.

Details on the derivation of continuum, monochromatic, and velocity maps from scanning FP observations are given in Fuentes-Carrera et al. (2004). In order to get a sufficient 
$\mathrm{S} / \mathrm{N}$ on the outer parts of each galaxy, we performed three spatial Gaussian smoothings $\left(\sigma=2.32^{\prime \prime}, 3.48^{\prime \prime}, 4.64^{\prime \prime}\right)$ on the resulting calibrated cube, so that a variable resolution radial velocity map was built using less spatially and spectrally smoothed pixels for regions with an originally higher $\mathrm{S} / \mathrm{N}$ and more spatially and spectrally smoothed pixels for the outer parts of the galaxy.

\section{Arp 240: NGC 5257 and NGC 5258}

\subsection{The pair of galaxies}

Arp 240 is an interacting galaxy pair consisting of two spiral galaxies: NGC 5257 and NGC 5258 (Fig. 1). Using the line-ofsight (LOS) radial velocities of the galaxies, corrected for the Local Group infall onto Virgo $\left(6798 \mathrm{~km} \mathrm{~s}^{-1}\right.$ and $6757 \mathrm{~km} \mathrm{~s}^{-1}$, for NGC 5257 and NGC 5258, respectively ${ }^{1}$ ), the galaxy pair is located at a distance of $\sim 100.0 \mathrm{Mpc}$. This implies linear diameters of $51.7 \mathrm{kpc}\left(106.6^{\prime \prime}\right)$ and $48.3 \mathrm{kpc}\left(99.6^{\prime \prime}\right)$, for NGC 5257 and NGC 5258, respectively, considering the $D_{25}$ values by de Vaucouleurs et al. (1991), and a projected separation between the two galaxies of $37.8 \mathrm{kpc}\left(78^{\prime \prime}\right)$. The general parameters of both galaxies are presented in Table 2 .

The pair belongs to the sample of bright Infrared Astronomical Satellite (IRAS) galaxies (Soifer et al. 1989). The total infrared luminosity $\left(L_{\mathrm{IR}}\right)$ of the system is $2.8 \times 10^{11} L_{\odot}$, and the total FIR luminosity equals $2.02 \times 10^{11} L_{\odot}$ (Sofue et al. 1993), with both galaxies contributing more or less equally to it. From their flux ratios, Liu \& Kennicutt (1995) classified both galaxies as starbursts. Simulations of this pair have been done by Holincheck et al. (2016) seeking to reconstruct the disturbed morphologies of interacting galaxies with the use of a restricted three-body simulation code, however no kinematical restrictions were considered for the modelling. The top panel of Fig. 1 shows the high spatial resolution Hubble Space Telescope (HST) image ${ }^{2}$ of the pair taken with the Advanced Camera for Surveys (ACS) in its Wide Field Channel (WFC) using the $\mathrm{F} 435 \mathrm{~W}$ filter (between the $U$ - and $B$ - bands). The middle panel of Fig. 1 shows the HST-ACS F814W (close to the $I$-band) image of the pair. Figure 2 shows the high-contrast $B$-band image of Arp 240 displaying the tidal features of each galaxy.

\subsection{NGC 5257}

NGC 5257 is classified as an SABb galaxy according to the HyperLEDA $^{3}$ database and as an SAB(rs)b pec HII LIRG galaxy in the $\mathrm{NED}^{4}$ database. General parameters of this galaxy are presented in Table 2. Top panel of Fig. 1 shows the western spiral arm is outlined by small bright "pearl-like" regions. In the middle panel of Fig. 1 a small bulge can be seen embedded in a faint bar $\sim 4^{\prime \prime}$ in length. Inner spiral arms are well-traced coming out of the bar. The external parts of both arms seem to be tidally stretched. From near-infrared (NIR) and $\mathrm{H} \alpha$ observations of this galaxy, Bushouse et al. (1998) found high levels of SF activity in the western arm of NGC 5257, while the nucleus shows a low degree of SF activity. A prominent stellar bar $\left(6.5^{\prime \prime}=3.1 \mathrm{kpc}\right.$, in length) can be seen in the $K$-band image by Bushouse et al.

\footnotetext{
1 Values taken from the LEDA database.

2 HST archive, proposal 10592 by Aaron Evans.

3 HyperLEDA database http://leda.univ-lyon $1 . \mathrm{fr} /$.

4 The NASA/IPAC Extragalactic Database (NED) is operated by the Jet Propulsion Laboratory, California Institute of Technology, under contract with the National Aeronautics and Space Administration.
}

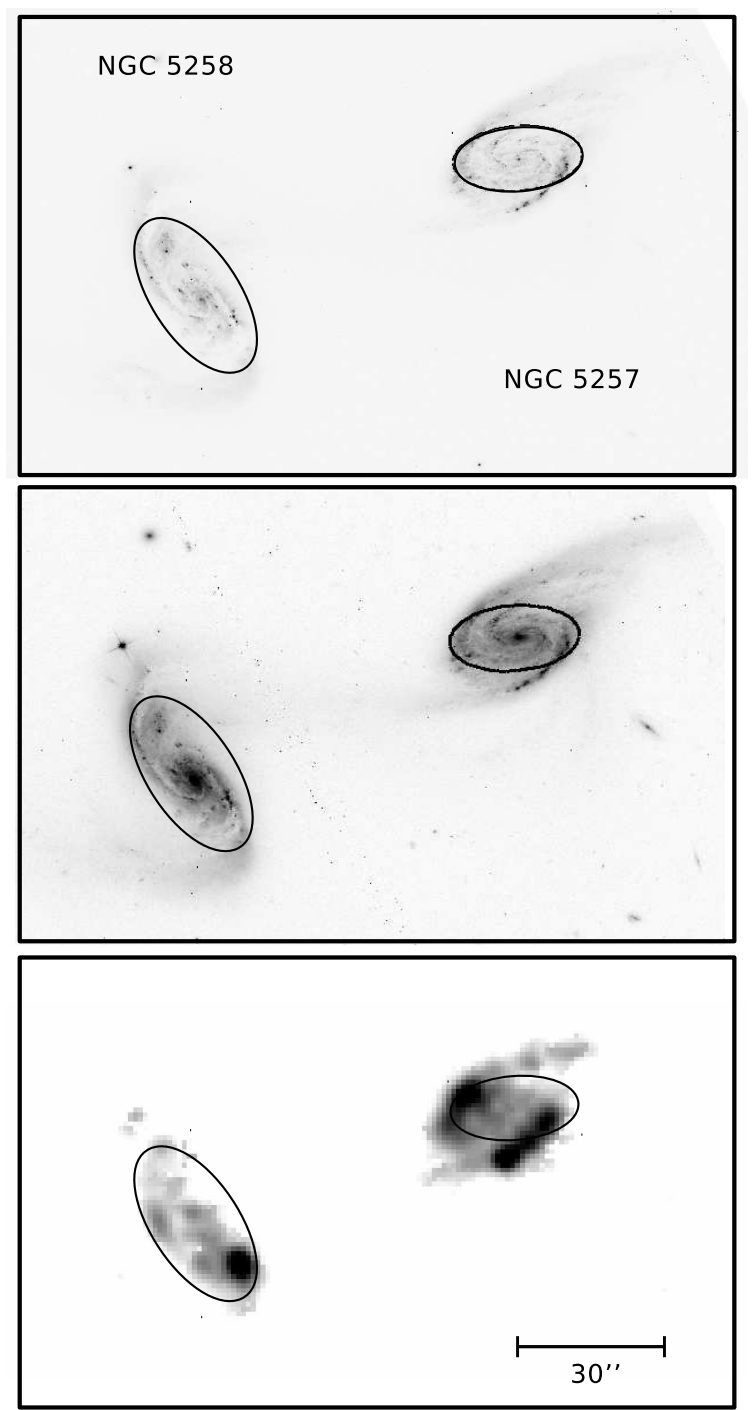

Fig. 1. Top panel: high spatial resolution HST image of Arp 240 taken with the Advanced Camera for Surveys (ACS) in its Wide Field Channel (WFC) in the F435W filter (between $U$ - and $B$-bands). Middle panel: HST-ACS WFC image in the F814W filter (close to the $I$-band). Bottom panel: monochromatic $\mathrm{H} \alpha$ (continuum subtracted) image of the pair obtained from the scanning Fabry-Perot interferometer PUMA data cubes. Ellipses indicate the location of the bifurcation radius, $R_{\mathrm{bif}}$, in the rotation curve of NGC 5257 and NGC 5258, respectively. These are discussed in Sect. 4.3. HST images taken from the HST archive, proposal 10592 by Aaron Evans. NGC 5257 is the galaxy on the right of the image, and NGC 5258 is the galaxy on the left. North is to the top, east is to the left.

(1998). Iono et al. (2005) studied the HI, radio continuum, and $\mathrm{CO}(1-0)$ emissions of this galaxy using the Very Large Array (VLA) and the Owens Valley Radio Observatory (OVRO) millimeter array, respectively. Figure 3 of their work shows a relatively undisturbed HI distribution for NGC 5257 but exhibits a $\mathrm{CO}(1-0)$ concentration near the nucleus extending to the northeast and to the south-west, forming a bar $10 \mathrm{kpc}$ long. Using the radio continuum fluxes, those authors estimated an SFR of $27.8 M_{\odot} \mathrm{yr}^{-1}$. Figure 2 shows two prominent tidal arms for this galaxy. The western arm is elongated becoming an apparent tidal bridge between both galaxies, while the eastern arm traces the beginning of a tidal tail. 
Table 2. General parameters of NGC 5257 and NGC 5258.

\begin{tabular}{|c|c|c|c|}
\hline Parameter & NGC 5257 & NGC 5258 & Reference \\
\hline \multirow[t]{2}{*}{ Coordinates $(\mathrm{J} 2000)^{a}$} & $\alpha=13^{\mathrm{h}} 39^{\mathrm{m}} 52.91^{\mathrm{s}}$ & $\alpha=13^{\mathrm{h}} 39^{\mathrm{m}} 57.70^{\mathrm{s}}$ & $\cdots$ \\
\hline & $\delta=+00^{\circ} 50^{\prime} 24.5^{\prime \prime}$ & $\delta=+00^{\circ} 49^{\prime} 51.1^{\prime \prime}$ & $\ldots$ \\
\hline \multirow{3}{*}{ Morphological type } & $\mathrm{SABb}^{a}$ & $\mathrm{SBb}^{a}$ & $\ldots$ \\
\hline & $\mathrm{SAB}(\mathrm{s}) \mathrm{b}$ pec HII $\mathrm{LIRG}^{b}$ & SA(s)b:pec; HII LINER ${ }^{b}$ & $\ldots$ \\
\hline & $\mathrm{SA}(\mathrm{rs}) \mathrm{b}$ pec & $\mathrm{SABb}$ pec & 1 \\
\hline Heliocentric systemic & $6800 \pm 4^{a, c}$ & $6782 \pm 9^{a, c}$ & $\ldots$ \\
\hline velocity $\left(\mathrm{km} \mathrm{s}^{-1}\right)$ & $6764^{d}$ & $6765^{d}$ & 2 \\
\hline Redshift $^{b}$ & 0.022676 & 0.022539 & $\ldots$ \\
\hline \multirow[t]{2}{*}{ Distance (Mpc) } & 98.0 & 97.4 & 3 \\
\hline & $100.3^{e}$ & $99.7^{e}$ & $\ldots$ \\
\hline Morphological position angle ${ }^{a}\left(^{\circ}\right)$ & 83.1 & 178 & $\ldots$ \\
\hline Morphological inclination $^{a}\left({ }^{\circ}\right)$ & 58 & 34 & $\ldots$ \\
\hline$m_{B}(\mathrm{mag})$ & 11.23 & 14.05 & 1 \\
\hline$m_{R}(\mathrm{mag})$ & 10.28 & 13.18 & 1 \\
\hline$m_{\text {Kext }}^{\mathrm{f}}(\mathrm{mag})$ & $10.084 \pm 0.047$ & $9.962 \pm 0.039$ & 4 \\
\hline$(B-I)$ & 1.59 & 1.47 & 1 \\
\hline$B_{T}^{0 g}$ & 11.03 & 13.90 & 1 \\
\hline$(B-V)_{T}^{0 h}$ & 0.51 & 0.42 & 1 \\
\hline$R_{25}=D_{25} / 2$ in $\operatorname{arcsec}(\mathrm{kpc})$ & $53.3(25.8)$ & $49.8(24.1)$ & 5 \\
\hline$R_{K 20}^{f}$ in $\operatorname{arcsec}(\mathrm{kpc})$ & $29.9(14.49)$ & $37.6(18.22)$ & 4 \\
\hline$R_{\mathrm{H} \alpha}^{i}$ in $\operatorname{arcsec}(\mathrm{kpc})$ & $17.98(8.71)$ & $18.04(8.75)$ & $\ldots$ \\
\hline \multicolumn{4}{|l|}{ Mean surface brightness } \\
\hline Within 25 isophote $\left(\mathrm{mag} / \mathrm{arcsec}^{-2}\right)$ & 22.74 & 23.26 & 1 \\
\hline$L_{\mathrm{H} \alpha}\left(10^{6} L_{\odot}\right)$ & 5 & 6 & 6 \\
\hline \multirow{2}{*}{$L_{\mathrm{IR}}\left(10^{11} L_{\odot}\right)$} & 1.7 & For the whole pair & 7 \\
\hline & 2.7 & For the whole pair & 8 \\
\hline \multirow[t]{4}{*}{$L_{\mathrm{FIR}}\left(10^{11} L_{\odot}\right)$} & 1.7 & $\ldots$ & 9 \\
\hline & 2.0 & For the whole pair & 6 \\
\hline & 1.5 & For the whole pair & 8 \\
\hline & 2.0 & For the whole pair & 10 \\
\hline$L_{\mathrm{IR}} / L_{B}$ & 12.0 & For the whole pair & 7 \\
\hline \multirow[t]{2}{*}{ Maximum rotation velocity $\left(\mathrm{km} \mathrm{s}^{-1}\right)$} & $248 \pm 10^{a}$ & $387 \pm 19^{a}$ & $\ldots$ \\
\hline & $270^{j}$ & $250^{j}$ & 2 \\
\hline Dynamical mass $\left(10^{11} M_{\odot}\right)$ & $0.28^{k}$ & $1.10^{k}$ & 2 \\
\hline \multirow[t]{2}{*}{ Mass in $\mathrm{HI}\left(\right.$ in $\left.10^{10} M_{\odot}\right)$} & 1.2 & 0.98 & 6 \\
\hline & 3.39 & For the whole pair & 2 \\
\hline Mass in $\mathrm{H}_{2}\left(\right.$ in $\left.10^{10} M_{\odot}\right)$ & 1.32 & 2.40 & 2 \\
\hline $\operatorname{SFR}^{l}\left(M_{\odot} \mathrm{yr}^{-1}\right)$ & 27.8 & 24.9 & 2,11 \\
\hline
\end{tabular}

Notes. ${ }^{(a)}$ HyperLEDA. ${ }^{(b)}$ NED. ${ }^{(c)}$ From radio observations. ${ }^{(d)}$ Median velocity of the CO(1-0) spectrum. ${ }^{(e)}$ From redshift value. ${ }^{(f)}$ 2MASS database $k \_m \_k 20 f e$ value. ${ }^{(g)}$ Total corrected magnitude in the RC3 system. ${ }^{(h)}$ Total corrected colour index in the RC 3 system. ${ }^{(i)}$ This work. ${ }^{(j)}$ From rotation curve with no inclination correction. ${ }^{(k)}$ Derived from the maximum radial extent of the CO(1-0) emission: $4.7 \mathrm{kpc}\left(0.18 R_{25}\right)$ for NGC 5257, $12 \mathrm{kpc}$ $\left(0.50 R_{25}\right)$ for NGC 5258 , and the observed rotational velocity divided by $\sin 90 .{ }^{(l)}$ From $L_{1.4 \mathrm{GHz}}$.

References. (1) Hernández-Toledo \& Puerari (2001); (2) Iono et al. (2005); (3) Mould et al. (2000); (4) (Skrutskie et al. 2006); (5) de Vaucouleurs et al. (1991); (6) Sofue et al. (1993); (7) Bushouse et al. (1998); (8) Dopita et al. (2002); (9) Casasola et al. (2004); (10) Georgakakis et al. (2000); (11) Yun et al. (2001).

\subsection{NGC 5258}

NGC 5258 is classified as an SBb galaxy in the HyperLEDA database and as an SA(s)b?pec HII low-ionization nuclear emission-line region (LINER) in the NED database. In their detailed analysis of the optical surface photometry of $(S+S)$ galaxies, Hernández-Toledo \& Puerari (2001) classified NGC 5258 as SABb pec. General parameters of this galaxy are presented in Table 2. The HST-ACS F435W image of this galaxy (top panel of Fig. 1) shows the bulge is surrounded by a ringlike structure. The northern arm of the galaxy bifurcates into a northern and western arm. This bifurcation is clearly marked by dust. This is also seen in the HST-ACS F814W image (middle panel of Fig. 1). A small bar almost aligned with the position angle, PA, of the galaxy is detected in this filter. Dust lanes trace part of the spiral arms. Important emission is seen along the southern arm in what seems to be a series of stellar clusters. These clusters are also seen in the F413W image. In their optical and NIR photometry analysis of interacting galaxies, Bushouse et al. (1998) found that the $\mathrm{H} \alpha$ emission map of this galaxy shows star-forming activity in the region south-west of the nucleus, coinciding with the $\mathrm{H} \alpha$ emission peak detected 


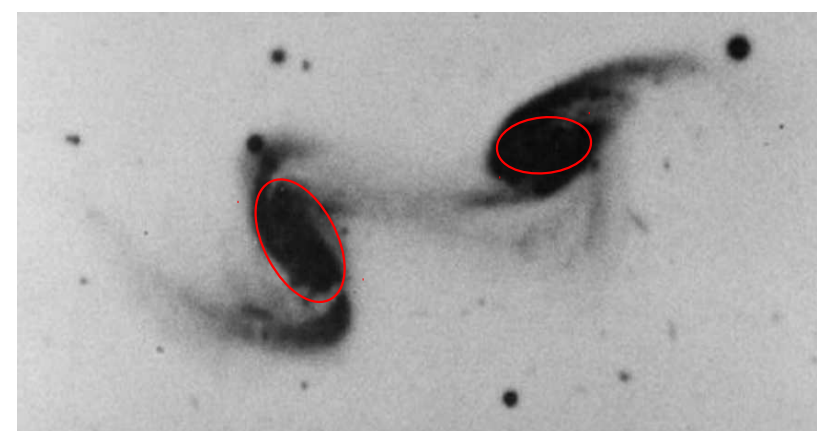

Fig. 2. High-contrast B image of Arp 240 showing the extension of the tidal features of each galaxy, taken from the "Catalog of Peculiar Galaxies" (Arp 1966). The ellipses indicate the location of the bifurcation radius, $R_{\mathrm{bif}}$, in the rotation curve of NGC 5257 and NGC 5258, respectively. These are discussed in Sect. 4.3.

by Dopita et al. (2002), while the nucleus itself shows low starforming activity. The VLA images by Iono et al. (2005) show an HI tail $\sim 125^{\prime \prime}(60 \mathrm{kpc})$ long, extending beyond the optical tail. The CO(1-0) emission detected by Iono et al. (2005) closely traces the optical morphology. Figure 2 shows that the northern extension of the northern arm of NGC 5258 appears to be truncated, while the western extension points towards the companion and seems to join it through a tidal bridge. The southern arm of the galaxy broadens and becomes an extended tidal tail.

\section{Kinematic results}

\section{1. $H_{\alpha}$ Image}

The bottom panel of Fig. 1 shows the $\mathrm{H} \alpha$ line intensity image of the pair derived from PUMA observations by integrating the monochromatic $\mathrm{H} \alpha$ profile in each pixel of the data cube. For NGC 5257, HII regions outline both arms, especially the western arm. For the latter, the same three HII regions detected in the $\mathrm{H} \alpha$ images by Bushouse et al. (1998) and Dopita et al. (2002) are seen. A prominent HII region is observed in the middle part of the eastern arm. The centre of the galaxy is devoid of HII regions. $\mathrm{H} \alpha$ emission is seen along the tidal tail and the beginning of the bridge between the two galaxies. For NGC 5258, a prominent HII region is seen in the south-western side of the galaxy, and much fainter HII regions are seen in the beginning of the northern arm. These regions are also seen in the $\mathrm{H} \alpha$ images by Bushouse et al. (1998) and Dopita et al. (2002). The tidal bridge visible in the high-contrast $B$-band image is not detected in $\mathrm{H} \alpha$.

\subsection{Velocity Fields}

Table 2 indicates that both galaxies are classified as barred or non-barred galaxies depending on the authors, from SA to SAB for NGC 5257 and from SA to SB for NGC 5258. Visual inspection of the HST F814W image makes plausible the existence of small bars of equal size in both galaxies, but slightly stronger for NGC 5258 than for NGC 5257. On the other hand, the bar is almost aligned with the major axis of NGC 5258, while it makes roughly an angle of $\pi / 4$ in the case of NGC 5257 .

The top panel of Fig. 3 shows the velocity field of both galaxies; the right panel of the same figure displays the corresponding isovelocities. The innermost parts of NGC 5257 exhibit isovelocities almost parallel to each other and that are not perpendicular to the major axis of the galaxy within a region that has an extension of $\sim 7.8^{\prime \prime}(3.8 \mathrm{kpc})$. This is related to the presence of a stellar bar oriented between the major and the minor axis of the galaxy as can be seen on the HST images and on the velocity field itself. For NGC 5258, the isophotes along the minor axis are nearly straight with velocities around $6780 \mathrm{~km} \mathrm{~s}^{-1}$. A little further away from the centre $\left(\sim 2.4 \mathrm{kpc}, 5^{\prime \prime}\right)$, the isovelocities on one side and the other of this axis display the expected "V" shape associated with differential rotation. The bar being aligned with the major axis, no asymmetrical distortions are expected in the isovelocities but a small stretching between the galaxy centre and the "V" shape is observed. As we go further out of the galaxy, the "V" shape of the isovelocities rotates slightly along the PA on both sides of the galaxy (at $\sim 6550 \mathrm{~km} \mathrm{~s}^{-1}$ for the northern side and at $\sim 6950 \mathrm{~km} \mathrm{~s}^{-1}$ for the southern side). This twist within the optical radius, an obvious sign of interaction, is especially seen on the northern side.

\subsection{Rotation curves}

In the case of non-merging systems (systems where the disc and the bulge of both galaxies are distinct and where the discs of both galaxies are separated by a distance larger than their optical diameter), velocity fields are usually rather smooth and symmetric, resulting in symmetric and low-scattered rotation curves (RCs) up to a certain radius (Fuentes-Carrera et al. 2004). With this assumption in mind, the RC of each galaxy was computed in order to obtain a symmetric curve and to minimize scatter on each side of the curve, leading to an optimal set of kinematical parameters. Our methodology is described in Fuentes-Carrera et al. (2004). In order to minimize the influence of non-circular velocity components that increase toward the minor axis of the $\mathrm{RC}$, both $\mathrm{RC}$ were computed considering points on the velocity field within an angular sector of $36^{\circ}$ on each side of the galaxy's position angle (PA).

Figure 4 shows the RCs of NGC 5257 and NGC 5258. The most symmetrical, smooth, and less-scattered RC, displaying a residual velocity field without systemic features (see Sect. 4.4.2), was derived for both galaxies. The kinematical parameters used to derive the RC of each galaxy are presented in Table 3. Error bars give the dispersion of the rotation velocities computed for all the pixels found inside each elliptical ring having a width of about $\sim 1.5^{\prime \prime}$ and defined by the successive bins. The presence of a bar induces non-circular motions in the central regions of the disc due to the streaming of the gas along the bar. If the bar is parallel to the major axis, as is the case for NGC 5258, the rotational velocities are underestimated in the central region of the galaxy (e.g. Dicaire et al. 2008; Randriamampandry et al. 2015). On the contrary, if the bar is perpendicular to the major axis, the measured rotational velocities are overestimated. For an intermediate case like NGC 5257, we do not expect any signature in the rotation curve. In our case, no correction has been applied to any of the RCs to take into account the effect of the bar, since this feature is fairly weak and small in both galaxies.

The following set of values was derived for NGC 5257: $\mathrm{PA}=(95 \pm 3)^{\circ}, i=(58 \pm 5)^{\circ}$, and $V_{\text {sys }}=(6812 \pm 5) \mathrm{km} \mathrm{s}^{-1}$. The kinematical centre of the galaxy lies $1.3^{\prime \prime}(\sim 630 \mathrm{pc})$ north of the photometric centre of the HST image in the F814W filter. However, this difference is not significant considering the seeing of our FP observations. Except for the innermost regions $\left(R<2.4 \mathrm{kpc}, 5^{\prime \prime}\right)$, the $\mathrm{RC}$ is symmetric up to a radius $R=17.0^{\prime \prime}$ $(8.2 \mathrm{kpc})$ where the rotation velocity reaches $V_{\text {rot }}=290 \mathrm{~km} \mathrm{~s}^{-1}$. At this radius, which we shall call $R_{\text {bif }}$, the curve bifurcates. The rotation velocity on the approaching side of the galaxy decreases, while for the receding side, the velocity slowly increases.

The RC of NGC 5258 was derived using the following set of values: $\mathrm{PA}=(218 \pm 5)^{\circ}, \quad i=(57 \pm 4)^{\circ}$, and 

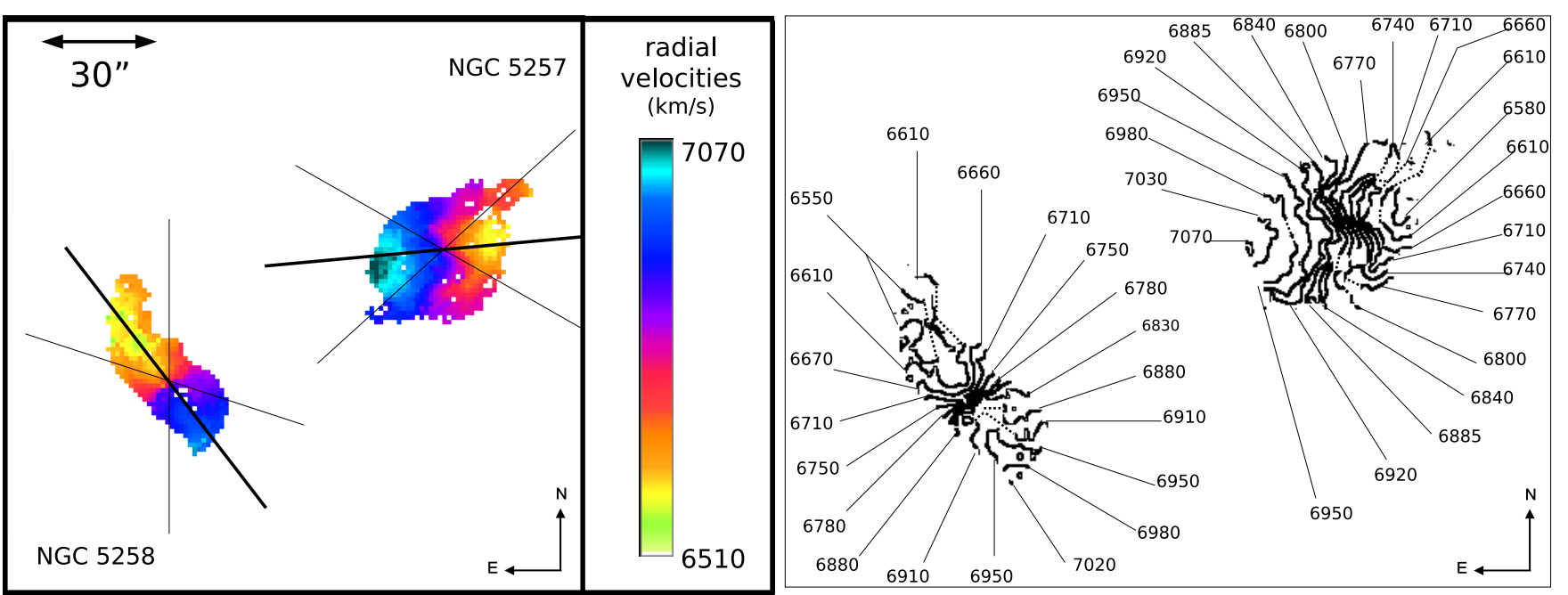

Fig. 3. Left panel: velocity field of Arp 240 (NGC 5257/58). The solid thick line indicates each galaxy's kinematic position angle (PA), the solid thin lines indicate the angular sectors from both sides of the major axis considered for the computation of each galaxy's rotation curve. Right panel: isovelocities plot for Arp 240 (NGC 5257/58).

$V_{\text {sys }}=(6762 \pm 5) \mathrm{km} \mathrm{s}^{-1}$. The kinematical centre of the galaxy lies $0.8^{\prime \prime}(\sim 390 \mathrm{pc})$ north-east of the photometric centre of the HST image in the F814W filter. Again, this difference is not significant considering the seeing of our FP observations. The RC is symmetric up to $R=16^{\prime \prime}(7.8 \mathrm{kpc})$, except for the points within the inner $3.0^{\prime \prime}(1.5 \mathrm{kpc})$. After that radius where $V_{\text {rot }} \sim$ $220 \mathrm{~km} \mathrm{~s}^{-1}$, the velocities on the receding side of the galaxy increase, while those on the approaching side remain fairly constant. We shall also call this radius $R_{\text {bif }}$. Table 4 summarizes the features of each galaxy discussed in Sects. 4.1-4.3.

\subsection{Non-circular motions}

\subsubsection{Velocity dispersions}

The gas velocity dispersion, $\sigma$, for each pixel was computed from the $\mathrm{H} \alpha$ velocity profiles after correction from instrumental and thermal widths, $\sigma_{\text {inst }}=\mathrm{FSR} /(\mathcal{F} \times 2 \sqrt{2 \ln 2})=16.4 \mathrm{~km} \mathrm{~s}^{-1}$ and $\sigma_{\text {th }}=9.1 \mathrm{~km} \mathrm{~s}^{-1}$, respectively. The latter value was estimated by assuming an electronic temperature of $T_{\mathrm{e}}=10^{4} \mathrm{~K}$ in the expression $\sigma_{\text {th }}=\left(k T_{\mathrm{e}} / m_{\mathrm{H}}\right)^{1 / 2}$. Assuming that all the profiles (instrumental, thermal, and turbulent) are described by Gaussians, the velocity dispersion was estimated using $\sigma=$ $\left(\sigma_{\text {obs }}^{2}-\sigma_{\text {inst }}^{2}-\sigma_{\text {th }}^{2}\right)^{1 / 2}$.

The top left panel of Fig. 5 shows the velocity dispersion map of NGC 5257. High velocity dispersion values ranging between $30 \mathrm{~km} \mathrm{~s}^{-1}$ and $55 \mathrm{~km} \mathrm{~s}^{-1}$ are seen along both spiral arms. The highest values match the location of the HII region in the eastern arm. Velocity values larger than $35 \mathrm{~km} \mathrm{~s}^{-1}$ are observed along the western spiral arm matching the location of the $\mathrm{H} \alpha$ maxima. Values of $\sim 25 \mathrm{~km} \mathrm{~s}^{-1}$ are observed on the tip of this arm coinciding with the beginning of the tidal arm. The top right panel of Fig. 5 shows the velocity dispersion map of NGC 5258. Large values $\left(\sim 55 \mathrm{~km} \mathrm{~s}^{-1}\right)$ are seen on the south-western side of the galaxy matching the location of the large HII region. The highest velocity dispersions are asymmetrically observed on the southern part of the galaxy.

\subsubsection{Residual velocity fields}

The residual velocity field of each galaxy was obtained by subtracting an axisymmetric velocity field model from the observed
NGC 5257: $H \alpha$ rotation curve

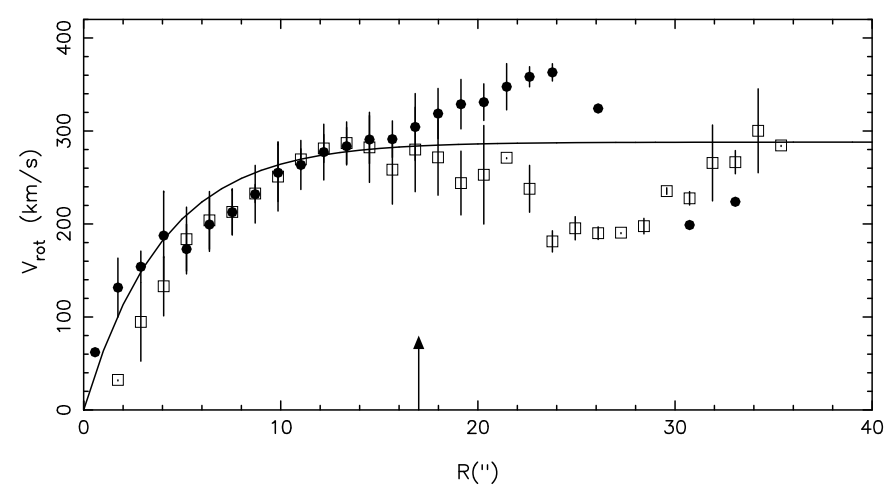

NGC 5258: $H \alpha$ rotation curve

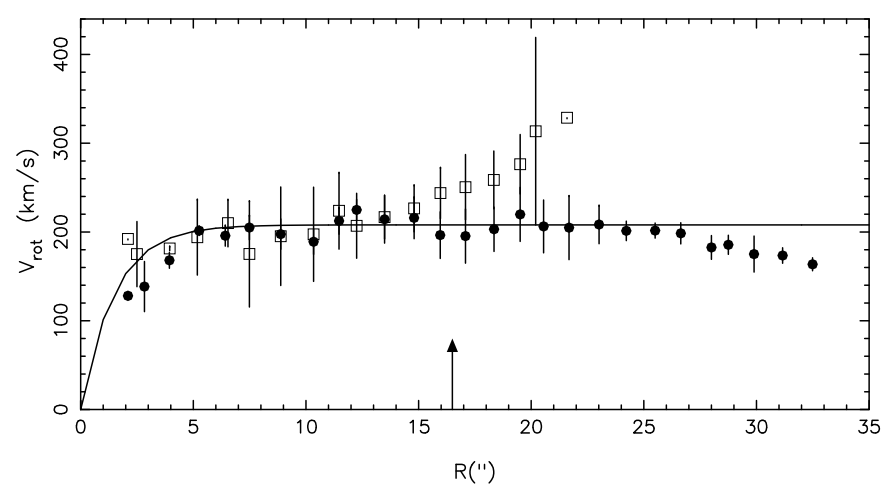

Fig. 4. Top panel: rotation curve (RC) of NGC 5257 derived from scanning Fabry-Perot (FP) observations of the ionized gas. Dark circles represent velocities on the receding side of the galaxy; light squares indicate velocities on the approaching side of the galaxy. Bottom panel: RC of NGC 5258. Dark circles represent velocities on the receding side of the galaxy; light squares indicate velocities on the approaching side of the galaxy. Solid lines show the parametric fit of each RC discussed in Sect. 4.4.2. Arrows indicate the position of $R_{\mathrm{bif}}$ discussed in Sect. 4.3.

velocity field. The model velocity field was derived by fitting the observed RC of the galaxy with a simple parametric function, $A \times\left(1-\mathrm{e}^{-r / B}\right)$, assuming it reflects pure circular motions. A check has been done on this image to ensure that its pattern 
Table 3. Kinematical parameters of NGC 5257 and NGC 5258 from this work.

\begin{tabular}{lll}
\hline \hline Parameter & NGC 5257 & NGC 5258 \\
\hline Heliocentric systemic velocity $\left(\mathrm{km} \mathrm{s}^{-1}\right)$ & $6812 \pm 5$ & $6762 \pm 5$ \\
PA $\left(^{\circ}\right)$ & $95 \pm 3$ & $218 \pm 5$ \\
Kinematical inclination $\left(^{\circ}\right)$ & $58 \pm 5$ & $57 \pm 4$ \\
$R_{\text {bif }}$ in $\operatorname{arcsec}(\mathrm{kpc})$ & $17(8.23)$ & $16(7.76)$ \\
$V_{\text {bif }}\left(\mathrm{km} \mathrm{s}^{-1}\right)$ & 290 & 220 \\
$\Omega_{\mathrm{p}}\left(\mathrm{km} \mathrm{s}^{-1} \mathrm{kpc}^{-1}\right)$ & $20 \pm 3$ & $\ldots$ \\
$R_{\text {cor }}$ in arcsec $(\mathrm{kpc})$ & $15 \pm 2(7.27 \pm 0.97)$ & $\cdots$ \\
& & \\
Maximum rotation velocity $\left(\mathrm{km} \mathrm{s}^{-1}\right)$ & $290^{a}$ & $210^{a}$ \\
& $360 \pm 10^{b}$ & $330 \pm 5^{b}$ \\
& $325^{c}$ & $320^{c}$ \\
\hline
\end{tabular}

Notes. ${ }^{(a)}$ From analytical fit of $\mathrm{H} \alpha \mathrm{RC}$ before bifurcation (Fig. 4). ${ }^{(b)}$ Point with highest velocity in $\mathrm{H} \alpha \mathrm{RC}$ considering all points (Fig. 6). ${ }^{(c)}$ From fit of multi-wavelength RC (Fig. 12).

Table 4. H $\alpha$ emission, velocity fields, and rotation curve features for NGC 5257 and NGC 5258.

\begin{tabular}{|c|c|c|c|c|}
\hline \multicolumn{5}{|c|}{$\mathrm{H} \alpha$ IMAGE } \\
\hline Galaxy & Prominent HII regions & Emission in central parts & Emission along tidal tail & Emission along tidal bridge \\
\hline NGC 5257 & YES -along both spiral arms & $\mathrm{NO}$ & YES & YES -at the beginning of the feature \\
\hline NGC 5258 & YES -only one & NO & NO & NO \\
\hline \multicolumn{5}{|c|}{ VELOCITY FIELD } \\
\hline Galaxy & Central isovelocities & Presence of bar (PA) & Outer isovelocities & Visual signs of interaction \\
\hline NGC 5257 & $\begin{array}{c}\text { Parallel to each other, } \\
\text { not perpendicular to major axis }\end{array}$ & YES & $\begin{array}{c}\text { Follow rotating disc } \\
\text { pattern }\end{array}$ & YES -on tidal tail \\
\hline NGC 5258 & $\begin{array}{l}\text { Parallel to each other, } \\
\text { perpendicular to major axis }\end{array}$ & YES & $\begin{array}{l}\text { Isovelocities "V" shape } \\
\text { rotates along PA }\end{array}$ & YES -along disc \\
\hline \multicolumn{5}{|c|}{ H $\alpha$ ROTATION CURVE } \\
\hline Galaxy & $\begin{array}{l}\text { Kinematic and Photometric } \\
\text { centres match }\end{array}$ & Inner asymmetries & Symmetrical & Bifurcated \\
\hline NGC 5257 & YES -within PUMA seeing & From $0^{\prime \prime}$ to $5^{\prime \prime}$ & From $5^{\prime \prime}$ to $17^{\prime \prime}$ & From $17^{\prime \prime}$ \\
\hline NGC 5258 & YES -within PUMA seeing & From $0^{\prime \prime}$ to $2.5^{\prime \prime}$ & From $2.5^{\prime \prime}$ to $16^{\prime \prime}$ & From $16^{\prime \prime}$ \\
\hline
\end{tabular}

does not contain any features due to a bad determination of the systemic velocity, kinematic centre, PA, and inclination as shown by Warner et al. (1973). The bottom left panel of Fig. 5 shows the residual velocities map for NGC 5257. A contribution of positive residual velocities $\left(\sim 40 \mathrm{~km} \mathrm{~s}^{-1}\right)$ is seen in the southcentral part of the galaxy; no symmetric counterpart is observed on the other side of the major axis of the galaxy. Values ranging from $40 \mathrm{~km} \mathrm{~s}^{-1}$ to $110 \mathrm{~km} \mathrm{~s}^{-1}$ are seen on the northern arm of the galaxy associated with the presence of the large HII regions. Positive residual velocities are seen on the tip of this arm. Negative values ranging between $-40 \mathrm{~km} \mathrm{~s}^{-1}$ and $-100 \mathrm{~km} \mathrm{~s}^{-1}$ follow the distribution of strong $\mathrm{H} \alpha$ emission along the southern arm. Negative values of $\sim-100 \mathrm{~km} \mathrm{~s}^{-1}$ are also observed on the tip of this arm to the south-east. For NGC 5258 (bottom right panel of Fig. 5), positive residual velocities ranging between $30 \mathrm{~km} \mathrm{~s}^{-1}$ and $63 \mathrm{~km} \mathrm{~s}^{-1}$ are found at the location of the large HII region on the south side of the galaxy. Negative residual velocities ranging from $-30 \mathrm{~km} \mathrm{~s}^{-1}$ to $-100 \mathrm{~km} \mathrm{~s}^{-1}$ are located on the southern tip of the galaxy and negative values of $\sim-60 \mathrm{~km} \mathrm{~s}^{-1}$ are also detected on the northern arm of the object.

\subsection{Kinematic versus morphological features}

Two-dimensional velocity fields of disc galaxies portray the motion of ionized gas all over the galaxy enabling us to match these motions with different morphological structures. One can determine to what extent the gas follows a circular motion around the centre of the galaxy or if there are important contributions from non-circular velocities in the radial, azimuthal, and vertical directions due to the presence of these structures or to external perturbations. For this purpose, we compared the monochromatic $\mathrm{H} \alpha$ image of each galaxy with its velocity map and its RC (Fig. 6).

For NGC 5257, the inner points of the RC show an asymmetry between the approaching and receding sides. This corresponds to part of the central bar (points A in the bottom panel of Fig. 6 and region A in top panel of the same figure). From these points onwards, both sides of the $\mathrm{RC}$ remain symmetric up to a $R=17^{\prime \prime}(8.2 \mathrm{kpc})$ reaching a rotational velocity of $290 \mathrm{~km} \mathrm{~s}^{-1}$ (point $\mathrm{B}$ in the $\mathrm{RC}$ ). This radius matches the location of $R_{\mathrm{bif}}$ defined in Sect. 4.3. For the approaching side the rotation velocity increases to $340 \mathrm{~km} \mathrm{~s}^{-1}$ at $R=24^{\prime \prime}$ (point D in the RC). This radius corresponds to the last points of the main disc of the galaxy on the receding side (region $\mathrm{D}$ in the bottom panel of Fig. 6). Finally, $V_{\text {rot }}$ drops to a value of $\sim 200 \mathrm{~km} \mathrm{~s}^{-1}$ at a radius of $\sim 32^{\prime \prime}$ (15.5 kpc) (point $\mathrm{E}$ in the RC). This corresponds to the tip of the extended western arm of the galaxy in $\mathrm{H} \alpha$ (region E). For the receding side of the $\mathrm{RC}$ (open squares in the bottom panel of Fig. 6), the velocity decreases as we approach the western edge of the main disc of the galaxy at $17^{\prime \prime}(8.2 \mathrm{kpc}$; ellipse B). In particular, this change seems to be associated with the strong HII 

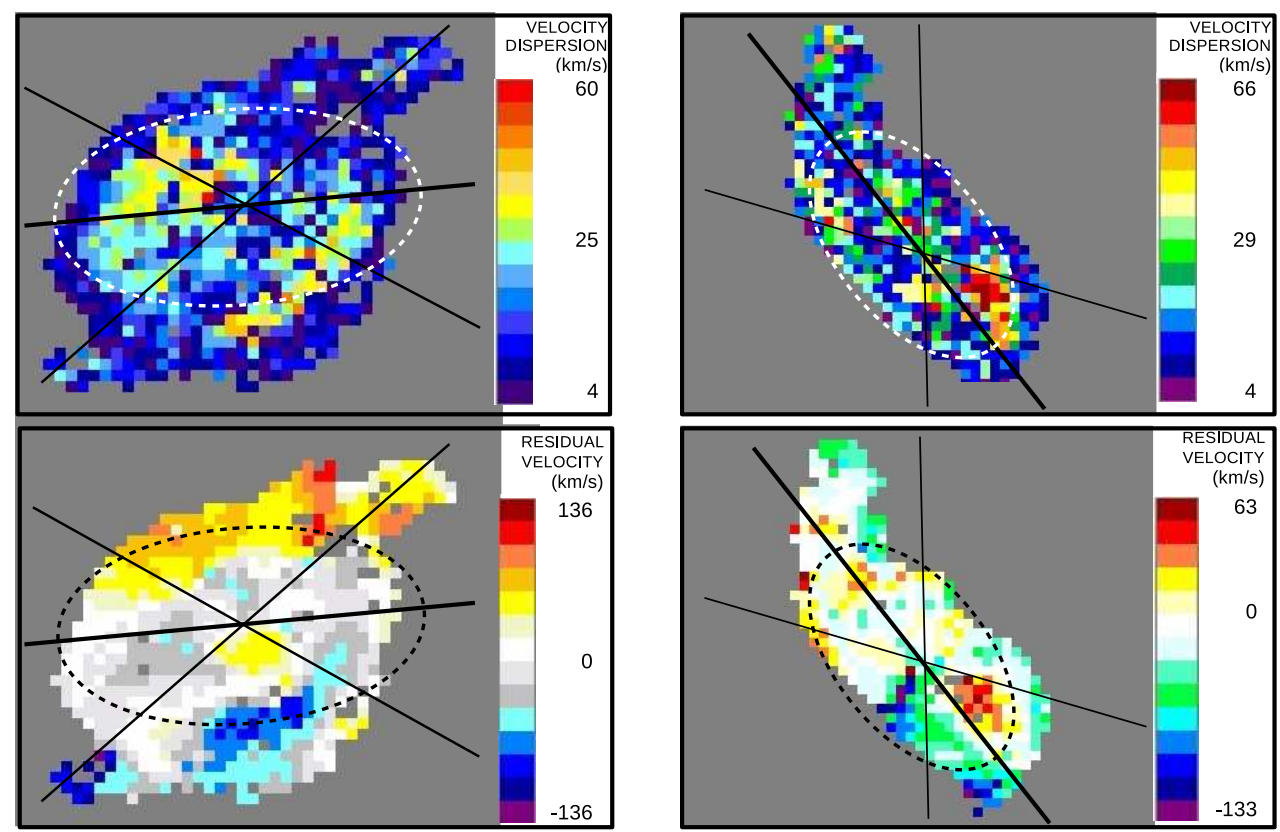

Fig. 5. Top left panel: velocity dispersion map for NGC 5257. Bottom left panel: residual velocities map of NGC 5257. Top right panel: velocity dispersion map for NGC 5258. Bottom right panel: residual velocities map of NGC 5258. The bold dark line is the PA and the fainter black lines the angular sector used to compute the RC of each galaxy. Ellipses indicate the location of the bifurcation radius, $R_{\mathrm{bif}}$, in the rotation curve of NGC 5257 and NGC 5258, respectively, discussed in Sect. 4.3. region marked with $\mathrm{F}$ in the top panel of Fig. 6 . The velocity $V_{\text {rot }}$ reaches a low-velocity plateau that seems to be associated with parts of the eastern tidal arm (region $\mathrm{G})$. Then it increases again from a radius of $30^{\prime \prime}(14.5 \mathrm{kpc})$ and seems to be associated with the tip of this tidal arm (region $\mathrm{H}$ ).

For NGC 5258, the asymmetry between the receding and approaching sides in the central parts corresponds to region $\mathrm{A}$ in Fig. 6. From a radius of $4^{\prime \prime}(1.9 \mathrm{kpc})$ to $16^{\prime \prime}(7.8 \mathrm{kpc}$; point $\mathrm{B}$ in the $\mathrm{RC}$ - bottom panel of Fig. 6), both sides are symmetric reaching a velocity of $220 \mathrm{~km} \mathrm{~s}^{-1}$. From this radius onwards, the velocity values increase for the receding side (open squares in the bottom panel of Fig. 6), while they remain fairly constant for the approaching side (dark circles in the same figure). This radius, $R_{\text {bif }}$, matches the edge of the main disc of the galaxy (ellipse B in the top panel of Fig. 6). For the receding side (open squares in bottom panel of Fig. 6), the velocity increases to $330 \mathrm{~km} \mathrm{~s}^{-1}$ at a radius of $12^{\prime \prime}(5.8 \mathrm{kpc}$; point $\mathrm{C}$ in the $\mathrm{RC})$. This corresponds to the southern tip of the galaxy where tidal effects along the arms of the galaxy begin to be seen (ellipse C). For the approaching side (dark circles in the bottom panel of Fig. 6), the velocity remains constant $\left(\sim 220 \mathrm{~km} \mathrm{~s}^{-1}\right)$ up to $R=23^{\prime \prime}(11.2 \mathrm{kpc})$, slightly decreasing from this radius to $27^{\prime \prime}$ (13.1 kpc; before region D in the bottom panel of Fig. 6). The velocity decreases along region $\mathrm{D}$ (top panel of Fig. 6), reaching a minimum value of $160 \mathrm{~km} \mathrm{~s}^{-1}$ at $32^{\prime \prime}(15.5 \mathrm{kpc}$; point E).

An ellipse with a major axis equal to $R_{\text {bif }}$ was plotted on the velocity dispersion and residual velocity maps of each galaxy. For NGC 5257, large values of velocity dispersion fall inside the ellipse for the eastern spiral arm; for the western arm, two of the HII regions fall inside the ellipse, while the brightest one falls outside the ellipse. Important values of the residual velocity along the spiral arms follow the ellipse, except for the values at the tips of the arms. For NGC 5258, both large dispersion velocity and residual velocity values probably associated with the large HII region fall inside the ellipse.

For both galaxies, we also plotted an ellipse with a major axis equal to $R_{\text {bif }}$ on images of the galaxy at different wavelengths (Fig. 7). The PA and inclination values of each ellipse were those used to derive the $\mathrm{RC}$ in Sect. 4.3. The position of the ellipse follows the location of $R_{\text {bif }}$ for each galaxy. For NGC 5257, the main disc of the galaxy is confined to the ellipse in the $K$-band, $4.5,8.0,12$, and $160 \mu \mathrm{m}$ images. For the optical images, only the southern part of the disc is limited by the ellipse. The maximum near ultraviolet (NUV) emission lies outside the ellipse, as well as the MIR and FIR maxima. For NGC 5258, the ellipse encompasses the ultraviolet (UV) emission from the main disc of the galaxy, while the tidally stretched arms lie outside the ellipse. For the NIR images, the main disc lies inside the ellipse. For the northern side of the galaxy, the ellipse matches the point where the northern arm bifurcates into the western and northern tidal extensions. The brightest HII regions on the southern side of the galaxy lie inside the ellipse.

\subsection{Pattern speed determination}

The pattern speed, $\Omega_{\mathrm{p}}$, is the speed of a density perturbation that moves along at a speed different from the speed of the objects within it. This density perturbation can be due to the presence of a bar, a spiral density wave, and/or the presence of a companion. The value of $\Omega_{\mathrm{p}}$ can be estimated from kinematic measurements without adopting any specific dynamical model: the TremaineWeinberg method (TW; Tremaine \& Weinberg 1984) requires only knowledge of the distribution of intensity and velocity of a component that reacts to the density wave and that obeys the continuity equation. Though it has been mainly used with the stellar component of disc galaxies, the applicability of the TW method to $\mathrm{H} \alpha$ emission has been shown in Hernandez et al. (2005), Fathi et al. (2007, 2009), and Gabbasov et al. (2009) for isolated galaxies. We assumed this method is still valid for slightly perturbed, non-merging interacting galaxies for which the ionized gas mostly traces the global kinematics of each galaxy, such as occurs in Arp 240. For this pair of galaxies, both the disc and the bulge are distinguishable and the velocity field of each galaxy resembles that of a rotating disc. They do not appear to be strongly perturbed and no common $\mathrm{HI}$ envelope is observed. Although tidal features are seen, they do not seem to have important effects in the inner parts of each galaxy. The validity of this assumption will be discussed in Sect. 7. 

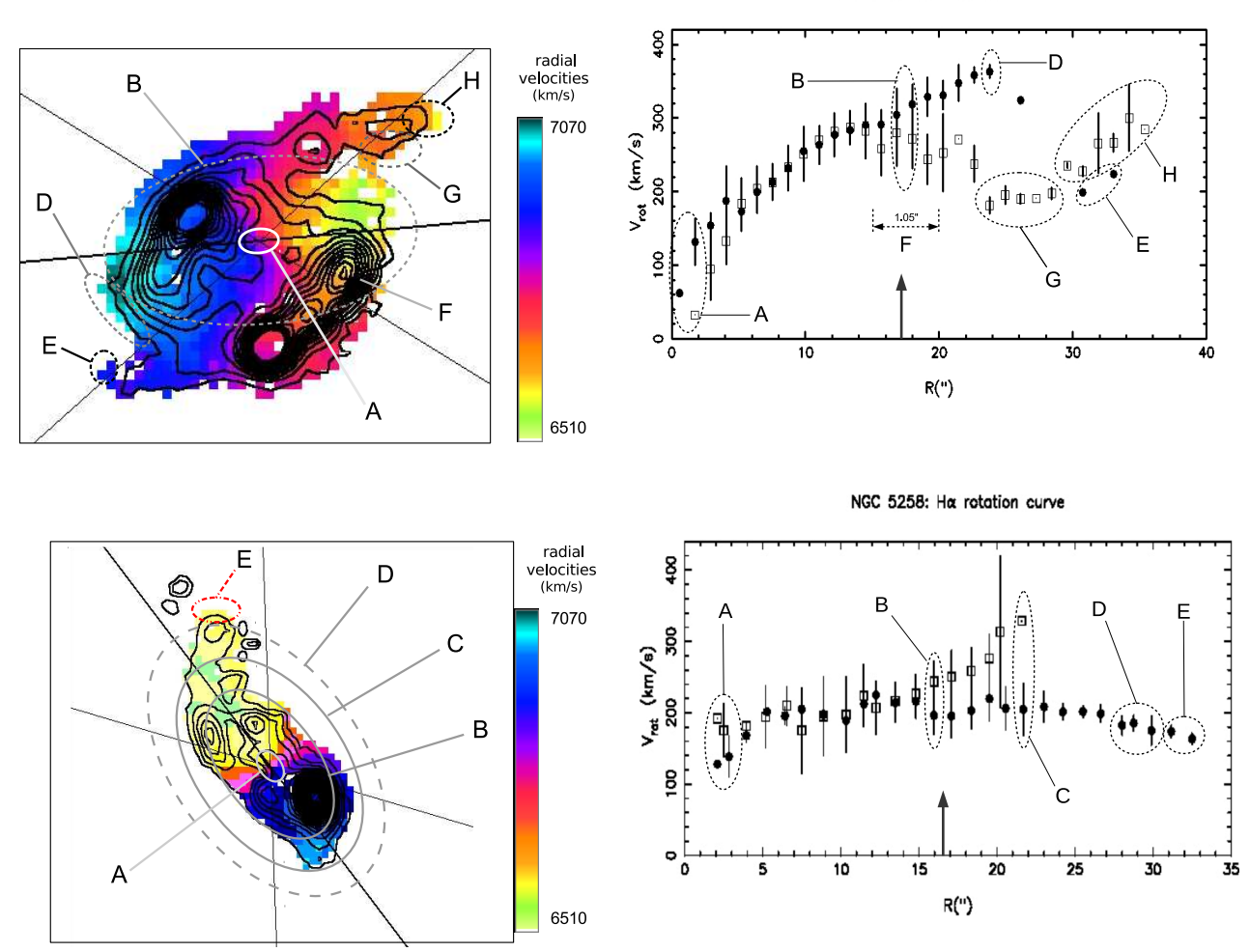

Fig. 6. Top left panel: velocity field of NGC 5257 with monochromatic isophotes. Letters indicate features associated with variations in the rotation curve of the galaxy. Solid line indicates the galaxy's position angle (PA), the slash-dotted lines indicate the angular sector from both sides of the major axis considered for the computation of the galaxy's RC. Top right panel: rotation curve (RC) of NGC 5257 derived from scanning Fabry-Perot (FP) observations of the ionized gas. Dark circles represent velocities on the receding side of the galaxy; light squares indicate velocities on the approaching side of the galaxy. The regions and letters shown are associated with features in the rotation curve of the galaxy. These are discussed in Sect. 4.5. Bottom left panel: same as top left panel for NGC 5258. Bottom right panel: RC of NGC 5258. Dark circles represent velocities on the approaching side of the galaxy; light squares indicate velocities on the receding side of the galaxy.
Using $\mathrm{H} \alpha$ emission as tracer, $\Omega_{\mathrm{p}}$ was derived from the relation (Merrifield \& Kuijken 1995)

$\Omega_{\mathrm{p}}=\frac{1}{\sin i} \frac{\langle V(x)\rangle}{\langle x\rangle}$,

where $\langle V(x)\rangle$ is the intensity-weighed, line-of-sight average velocity and $\langle x\rangle$ the intensity-weighted average position of the tracer along an "aperture" that is parallel to the apparent major axis of the galaxy. The pattern speed value $\Omega_{p}$ is obtained by fitting a straight line to these points. The slope of this line equals $\Omega_{\mathrm{p}} \times \sin i$. We set the origin of the Cartesian coordinate system at the derived kinematical centre of each galaxy and extracted the line-of-sight velocity along successive slits parallel to the major axis in twopixel intervals. The derived velocities within each simulated slit were "collapsed" into one single averaged value, $\langle V(x)\rangle$.

For NGC 5257, twenty averaged points were derived. The intensity-weighted average position per slit is shown in the left panel of Fig. 8. The five upper averaged points in the plot and the four lower averaged points were derived using considerably less points along the slit than for the averaged points on the main disc of the galaxy, plus they seem to be associated with the beginning of the tidal bridge and the tidal tail of the galaxy. For this reason, they were not considered for the computation of $\Omega_{\mathrm{p}}$. Also, since we are interested in deriving $\Omega_{\mathrm{p}}$ for a density perturbation (if any) on the main disc of the galaxy, we did not consider the two innermost averaged points since they are most likely associated with the kinematics of the bar. Once this preselection was done, a line was fitted to these points using linear least-squares (bottom right panel of Fig. 8). The slope of this line equals $\Omega_{\mathrm{p}} \times \sin i$. Considering the inclination value given in Sect. 4.3 , we find $\Omega_{\mathrm{p}}=20 \pm 3 \mathrm{~km} \mathrm{~s}^{-1} \operatorname{arcsec}^{-1}$. The error computation was done considering the error on the inclination, on the mean velocity, on the mean position along the line of nodes, and on the PA For NGC 5258, the $\mathrm{H} \alpha$ emission is mostly confined to the strong SF event on the southern arm of the galaxy, therefore it cannot be considered a good kinematical tracer and the TW method cannot be used to estimate $\Omega_{\mathrm{p}}$.

The value of the derived pattern speed for NGC 5257 was compared to the motion of the ionized gas in that galaxy. Figure 9 shows the $\mathrm{H} \alpha$ angular velocity curve for each side of NGC 5257. Horizontal dashed lines indicate the value of the derived pattern speed. The radius at which the pattern speed and the RC intersect gives the position of the corotation radius of the galaxy, $R_{\mathrm{cor}}$. This velocity matches the velocity of the ionized gas where the $\mathrm{RC}$ bifurcates at $(15 \pm 2)^{\prime \prime}$, while the position of the stellar bar matches the point where the RC becomes symmetric.

\section{Dynamical results}

\subsection{Multi-wavelength rotation curve}

Along with the $\mathrm{H} \alpha$ kinematic information from our FP observations, we used $\mathrm{CO}(1-0)$ and $\mathrm{HI}$ observations by Iono et al. (2005) in order to build a multi-wavelength rotation curve for each galaxy. In order to compare the different curves for both galaxies, we plotted the rotation velocities of Iono et al. (2005) RCs using the inclination of the optical RC (see Sect. 4.3). Figure 10 shows the resulting multi-wavelength rotation curves for both galaxies. For NGC 5257, the RCs superpose within the error bars up to $R=20^{\prime \prime}(9.7 \mathrm{kpc})$ with $V_{\text {rot }} \sim 300 \mathrm{~km} \mathrm{~s}^{-1}$. However, at larger radii, the optical RC decreases abruptly to $V_{\text {rot }} \sim 200 \mathrm{~km} \mathrm{~s}^{-1}$ from $24^{\prime \prime}(11.6 \mathrm{kpc})$ to $30^{\prime \prime}(14.5 \mathrm{kpc})$, then it increases to $300 \mathrm{~km} \mathrm{~s}^{-1}$ at $R=35^{\prime \prime}(17.0 \mathrm{kpc})$. Due to the beam size of the observations, no HI points are observed between $20^{\prime \prime}(9.7 \mathrm{kpc})$ and $36^{\prime \prime}$ $(17.5 \mathrm{kpc})$. The HI RC declines in velocity $\left(\Delta V>100 \mathrm{~km} \mathrm{~s}^{-1}\right)$ at large radii $\left(>31.5^{\prime \prime}=15.3 \mathrm{kpc}\right)$. According to Iono et al. (2005), this could be due to a significant warp in the outer parts of the $\mathrm{HI}$ disc, but we think that the decreasing RC is more likely due to streaming motions related to the interaction rather than circular motions out of the plane of the disc. For NGC 5258, the optical and $\mathrm{CO}(1-0) \mathrm{RCs}$ superpose smoothly within the error bars. 

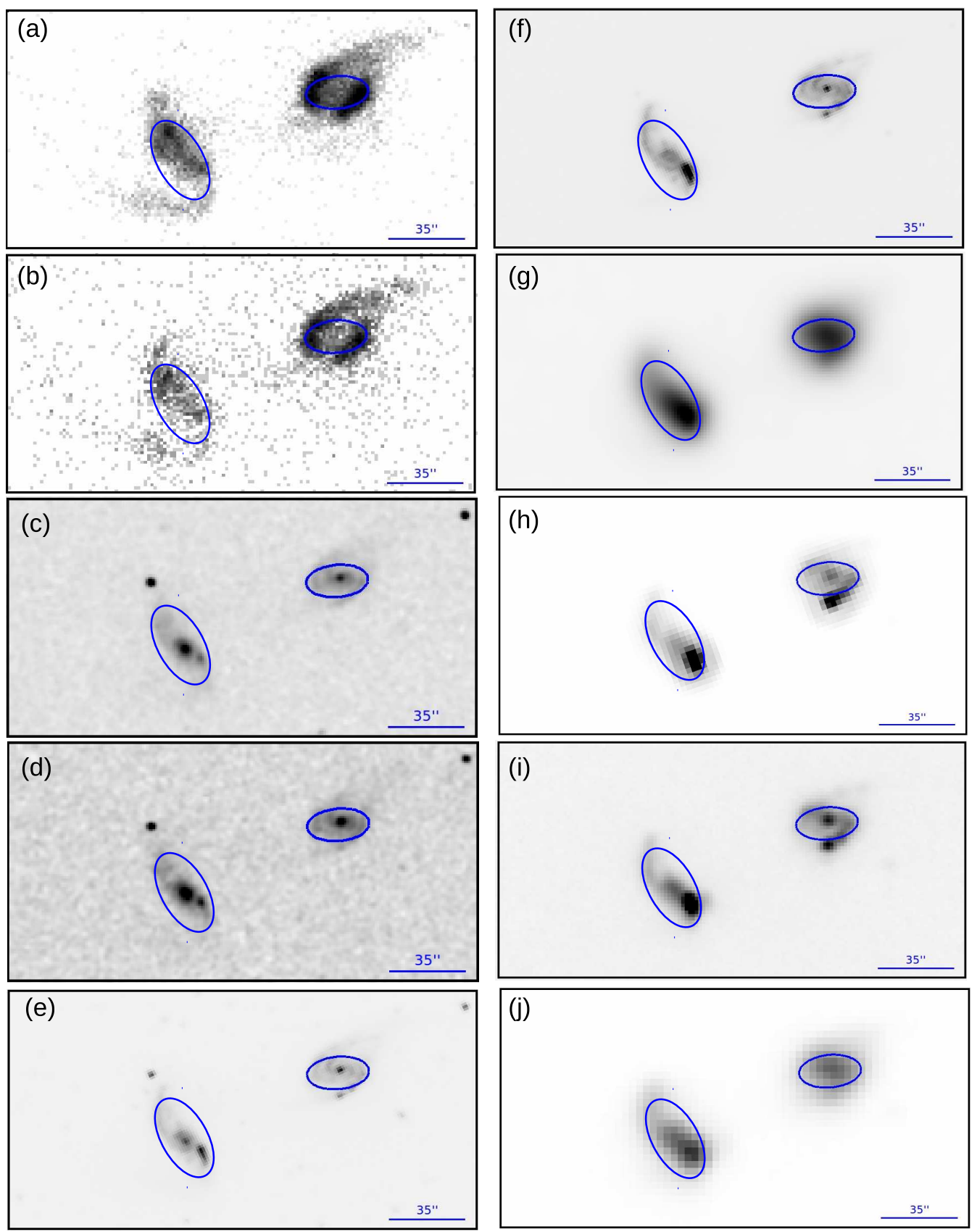

Fig. 7. Near-ultraviolet (panel $a$ ) and far-ultraviolet (panel $b$ ) images of Arp 240 taken from GALEX archive. The $J$ image (panel $c$ ) and $K$ image (panel d) from 2MASS (Skrutskie et al. 2006). The $4.5 \mu \mathrm{m}$ (panel e) and $8 \mu \mathrm{m}$ (panelf) images taken with Spitzer-IPAC camera (Smith et al. 2007); $12 \mu \mathrm{m}$ (panel g) image taken with WISE-PAC (Wright et al. (2010); IRSA catalogue); $24 \mu \mathrm{m}$ (panel $h$ ) image taken with Spitzer-MIPS (Smith et al. 2007); $60 \mu \mathrm{m}$ (panel i) and $160 \mu \mathrm{m}$ (panel j) images taken with Herschel "blue" channel and "red" channel, respectively (Proposal ID KPGT esturm 1). Ellipses indicate the location of the bifurcation radius, $R_{\mathrm{bif}}$, in the rotation curve of NGC 5257, discussed in Sects. 4.3, 4.5, and 4.6. North is to the top, east is to the left.

Both the optical and $\mathrm{CO}(1-0) \mathrm{RC}$ begin to decrease at $R=20^{\prime \prime}$ $(9.7 \mathrm{kpc})$; at $R=40^{\prime \prime}(19.4 \mathrm{kpc})$ the HI curve shows a large oscillation, from $260 \mathrm{~km} \mathrm{~s}^{-1}$ to $200 \mathrm{~km} \mathrm{~s}^{-1}$ and back, in a $60^{\prime \prime}(29.1 \mathrm{kpc})$ interval. After $R=100^{\prime \prime}(48.5 \mathrm{kpc})$, the HI curve remains constant at $250 \mathrm{~km} \mathrm{~s}^{-1}$.

\subsection{Mass estimates through rotation curves}

Making use of the possibility to disentangle circular from noncircular velocities in the optical RC, the multi-wavelength RCs were "cleaned" of points associated with non-circular motions (see Sect. 4.5) and points associated with features in the outer parts of the HI discs (Sect. 5.1). For NGC 5257, we removed the outer points of the averaged $\mathrm{H} \alpha \mathrm{RC}$ lying after the bifurcation radius, $R_{\text {bif }}$. For the HI points, we removed the last two points of the RC of Iono et al. (2005), which correspond to the decreasing part of the RC. For NGC 5258, we removed all points in the optical curve lying outside a radius of $\sim 20^{\prime \prime}=9.7 \mathrm{kpc}$ where the averaged $\mathrm{H} \alpha \mathrm{RC}$ starts to decrease. We also removed the $\mathrm{CO}(1-0)$ points lying beyond that radius since they showed an abrupt decrease in velocity. For the HI points, we removed points beyond $40^{\prime \prime}$ $(19.4 \mathrm{kpc})$ since they correspond to the beginning of the extended 

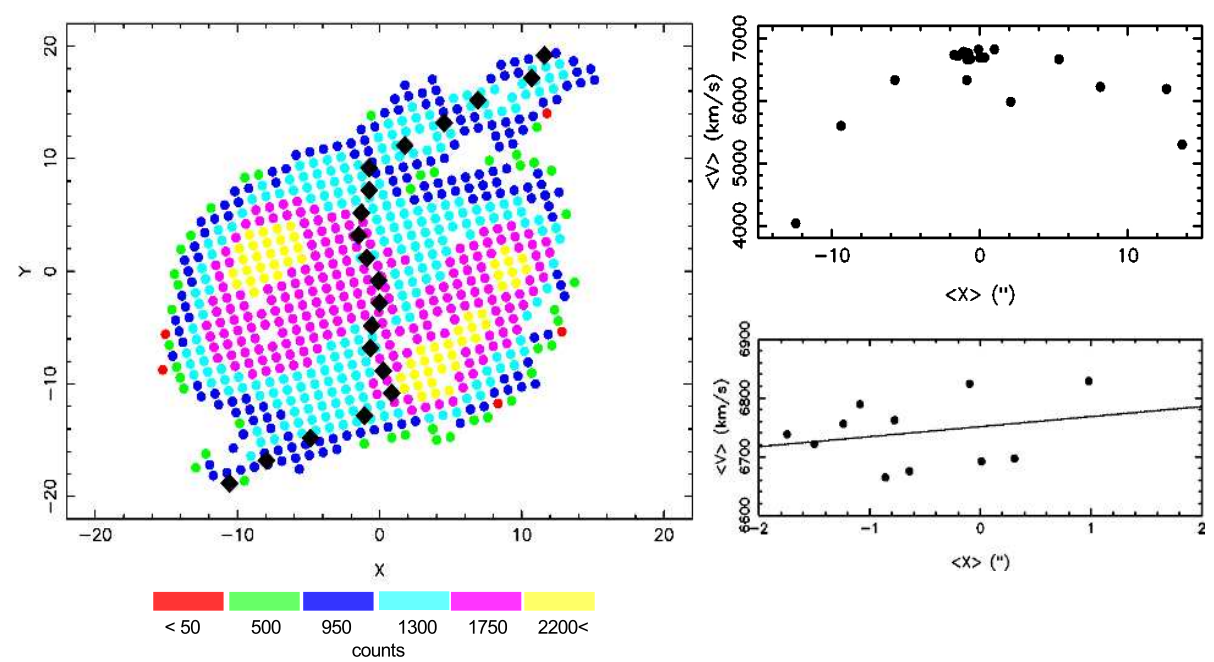

Fig. 8. Left panel: mean $Y$-position versus mean $X$-position for the determination of the pattern speed of NGC5257 using the Tremaine-Weinberg (TW) method. The galaxy has been rotated so that the PA lies along the $X$ axis. Dotted colours trace the monochromatic $(\mathrm{H} \alpha)$ emission of the galaxy. Top right panel: $\langle V(x)\rangle$ versus $\langle x\rangle$ for NGC 5257. Bottom right panel: zoom of the $\langle V(x)\rangle$ versus $\langle x\rangle$ plot for NGC 5257. The variables $\langle V(x)\rangle$ and $\langle x\rangle$ are described in Sect. 4.6.

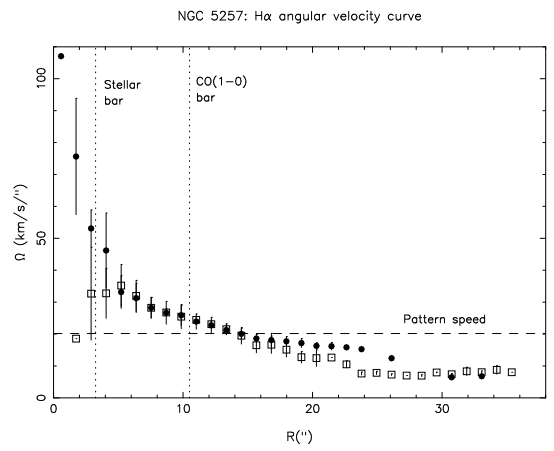

Fig. 9. Angular velocity for NGC 5257. Indicated on the figure are the location of both the stellar and $\mathrm{CO}(1-0)$ bar, as well as the pattern speed of the disc of the galaxy derived in Sect. 4.6.

HI tidal tail detected in the eastern side of the galaxy (Fig. 3 in Iono et al. 2005). Using these composite "clean" RCs, a range of possible masses was computed for each galaxy using the method by Lequeux (1983) according to which the total mass of a galaxy within a radius $R$ lies between 0.6 (the case of a disc-like mass distribution) and $1.0 \times\left(\mathrm{RV}_{\max }^{2}(R) / G\right)$ (the case of a spheroidal mass distribution). For NGC 5257, we considered the rotation velocity at the HI point before the velocity decreases $\left(V=270 \mathrm{~km} \mathrm{~s}^{-1}\right)$ to estimate the mass within $R=20^{\prime \prime}=9.7 \mathrm{kpc}=0.37 R_{25}$. The range of masses within this radius equals 0.9 to $1.6 \times 10^{11} M_{\odot}$. For NGC 5258, a rotation velocity of $250 \mathrm{~km} \mathrm{~s}^{-1}$ was considered to estimate the mass within the last HI point before the extended tidal tail, $R=38^{\prime \prime}=18.4 \mathrm{kpc}=0.72 R_{25}$. The range of masses within this radius is $1.6-2.7 \times 10^{11} M_{\odot}$. For the sake of comparison, we computed the mass ratio of the galaxies within the same radius in $D_{25} / 2$ units. The mass ratio, $M_{\mathrm{NGC} 5257} / M_{\mathrm{NGC} 5258}$, within a radius of $0.37 R_{25}$ goes from 0.5 to 1.6. On average, $M_{\mathrm{NGC} 5257} / M_{\mathrm{NGC} 5258} \sim 0.9$ within a radius of $0.37 R_{25}$. These results are shown in Table 5.

\subsection{Mass distribution and dark matter halo profile}

The mass distribution in each galaxy was estimated using the mass model described in Blais-Ouellette et al. (2001), which considers both the light distribution of the galaxy (stars and HI) and a theoretical dark halo profile to compute a $\mathrm{RC}$ that best fits the observed one. The mass-to-light ratio of the disc, $(M / L)_{\text {disc }}$, and of the bulge, $(M / L)_{\text {bulge }}$, the dark matter (DM) halo central density, $\rho_{0}$,

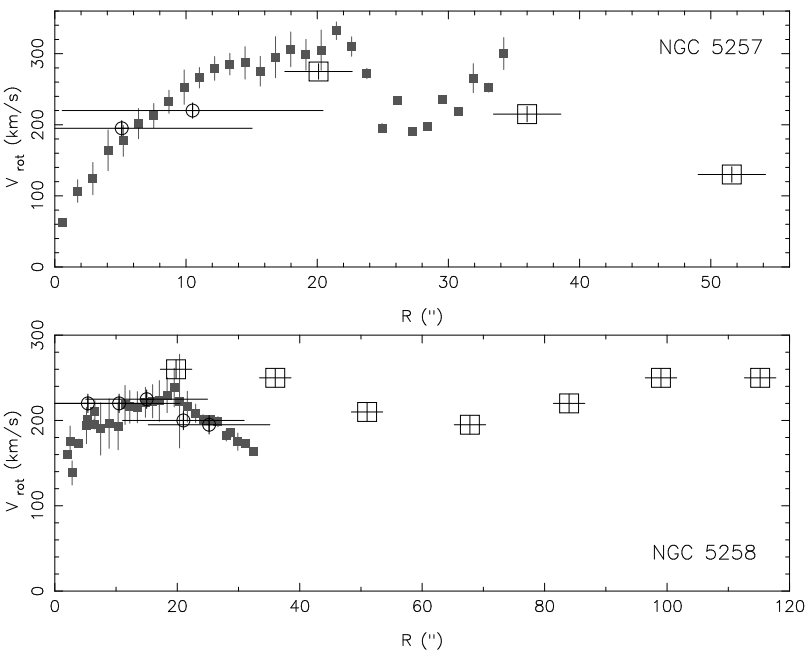

Fig. 10. Multi-wavelength rotation curves of NGC 5257 (top panel) and NGC 5258 (bottom panel) displaying all observed points. Small squares in the inner parts of the curve correspond to optical Fabry-Perot $\mathrm{H} \alpha$ observations. Empty circles in the inner parts correspond to the $\mathrm{CO}(1-$ 0 ) curve derived by Iono et al. (2005). Empty squares in the outer parts correspond to the HI curve also derived by Iono et al. (2005).

and core radius, $R_{0}$, are free parameters. In order to determine the light distribution from each galaxy, we derived the surface brightness (SB) profile of each galaxy from the F814W HST images using the STSDAS package ISOPHOTE in IRAF ${ }^{5}$. The light distribution was fitted with an exponential profile and a Sersic profile (Sersic 1968) in order to determine the contribution of the bulge and that of the stellar disc. Both profiles show the presence of the large HII regions in both galaxies. For NGC 5257, the northern HII region is responsible for the surface brightness increase around $12^{\prime \prime}$. The HI surface density distribution was taken from Iono et al. (2005). Figure 11 shows the SB profile of NGC 5257 (top) and of NGC 5258 (bottom).

Two types of DM halos were considered: a pseudo-isothermal sphere (pISO; Begeman 1987) and a Navarro-Frenk-White (NFW) profile (Navarro et al. 1996). Both a maximal and a

5 IRAF is distributed by the National Optical Astronomy Observatories, operated by the Association of Universities for Research in Astronomy, Inc., under cooperative agreement with the National Science Foundation. 
Table 5. Mass estimates for NGC 5257 and NGC 5258.

\begin{tabular}{lll}
\hline \hline Inside $R=0.37 D_{25} / 2$ & NGC 5257 & NGC 5258 \\
\hline$R=0.37 D_{25} / 2$ in arcsec $(\mathrm{kpc})$ & $20.0(9.70)$ & $18.4(8.94)$ \\
$V$ at $0.37 D_{25} / 2\left(\mathrm{~km} \mathrm{~s}^{-1}\right)$ & 270 & 290 \\
Dynamical mass $\left(10^{11} M_{\odot}\right)$ & $0.9-1.6^{a}$ & $1.5-2.5^{a}$ \\
& $1.48^{b}$ & $1.02^{b}$ \\
$M_{\text {disc }}{ }^{b}\left(10^{11} M_{\odot}\right)$ & 0.358 & 0.238 \\
$M_{\text {halo }}{ }^{b}\left(10^{11} M_{\odot}\right)$ & 1.12 & 0.779 \\
Mass ratio $\left(M_{\mathrm{NGC} 5257} / M_{\mathrm{NGC} 5258}\right)$ & & $0.5-1.6^{a}$ \\
& & $1.45^{b}$ \\
\hline \hline Inside furthest HI point on RC & NGC 5257 & NGC 5258 \\
before velocity decrease, $R_{\mathrm{HI}}$ & & $38.0\left(0.72 D_{25} / 2\right)$ \\
\hline$R_{\text {max }}$ in arcsec & $20.0\left(0.38 D_{25} / 2\right)$ & 250 \\
$V$ at $R_{\mathrm{HI}}\left(\mathrm{km} \mathrm{s}^{-1}\right)$ & 270 & $1.6-2.7^{a}$ \\
Dynamical mass $\left(10^{11} M_{\odot}\right)$ & $0.9-1.6^{a}$ & $2.87^{b}$ \\
& $1.48^{b}$ & 0.406 \\
$M_{\text {disc }}{ }^{b}\left(10^{11} M_{\odot}\right)$ & 0.358 & 2.46 \\
$M_{\text {halo }}{ }^{b}\left(10^{11} M_{\odot}\right)$ & 1.12 & \\
\hline
\end{tabular}

Notes. ${ }^{(a)}$ Using Lequeux (1983) method. ${ }^{(b)}$ From RC decomposition.

non-maximal disc were considered ${ }^{6}$. The model was fitted to the "cleaned" multi-wavelength $\mathrm{RC}^{7}$ of each galaxy described in Sect. 5.2. Table 6 shows the mass model parameters for each fit.

In our case, all halo models (with or without maximal disc) give similar $\chi^{2}$ values. However, $M / L$ values vary from one fit to the other -see Table 6 . We used these values to choose a "best" fit considering the multi-wavelength images of each galaxy discussed in Sect. 4.5. For NGC 5257, the best fit was achieved considering $(M / L)_{I}$ values of 3.5 for both the disc and the bulge. For this fit, a pISO halo and a maximal disc (with an important contribution of the bulge) were considered. For the other choices of DM halo and maximal or non-maximal disc, $(M / L)_{I}$ values are either low for both the disc and the bulge components or extremely high for the disc and extremely low for the bulge (see Table 6).

For NGC 5258, the best fit was achieved considering $(M / L)_{I}$ values of 3.2 for the bulge and 3.1 for the disc. In this case, a pISO halo and a maximal disc were considered. A pISO halo and maximal disc option result in low values for $(M / L)_{I}$. An NFW halo and a maximal disc give large values for $(M / L)_{I}$; while an NFW halo with a non-maximal disc result in low $(M / L)_{I}$ values for both the bulge and the disc. Values are shown in Table 6.

\section{Spectral energy distribution}

Figure 13 shows the SED of NGC 5257 (top panel) and of NGC 5258 (bottom panel) using the photometry values from Brown et al. (2014). Far-ultraviolet and NUV fluxes are taken from the Galaxy Evolution Explorer (GALEX), $u-, g_{-}, r-, i^{-}$, $z$-band fluxes from the Sloan Digital Sky Survey (SDSS), $J$-, $H$-, $K s$-band fluxes from the Two Micron All-Sky Survey (2MASS), along with Spitzer Space Telescope Infrared Array Camera (SST-IRAC) 3.6, 4.5, 5.8, 8 micron fluxes, Wide-field Infrared Survey Explorer (WISE) 3.6, 4.6, 12, 22 micron fluxes, and IRAS 60 and 100 micron fluxes.

\footnotetext{
6 We considered the "maximal disc" definition of Sackett (1997) for which $85 \% \pm 10 \%$ of the total rotational support of the galaxy at a radius $2.2 \times$ scale radius is contributed by the stellar disc mass component.

7 For both galaxies, the innermost points of the $\mathrm{CO}(1-0) \mathrm{RC}$ were removed due to their large position error bars.
}
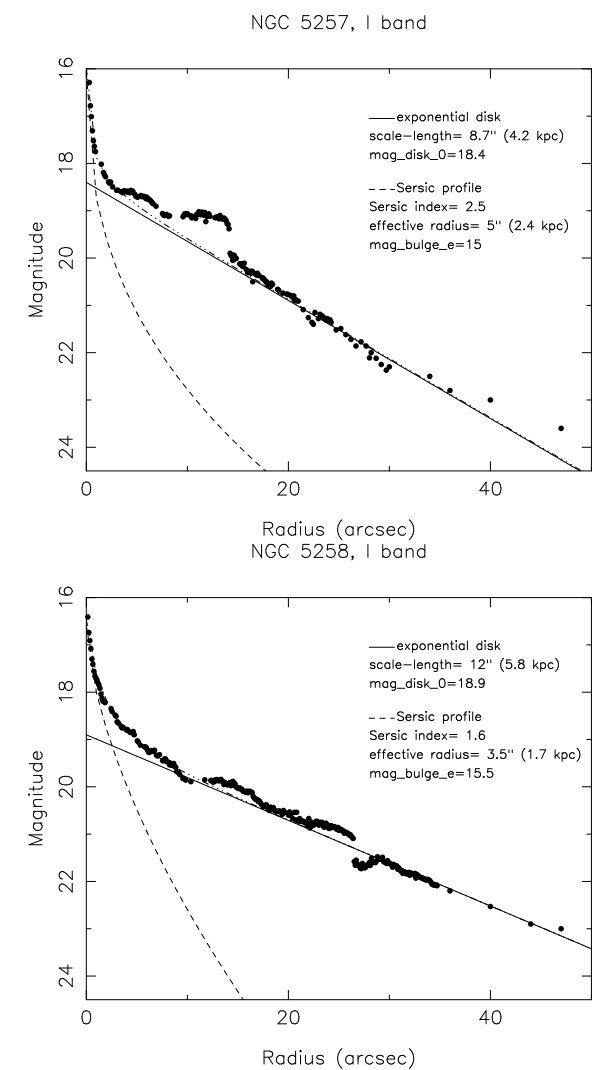

Fig. 11. Top panel: surface brightness profile of NGC 5257 in the HSTACS F814W filter. Image taken from the HST archive, proposal 10592 by Aaron Evans. Bottom panel: surface brightness profile of NGC 5258 in the HST-ACS F814W filter. Profiles have been decomposed in an exponential disc and a Sersic bulge component. The dashed-dotted line shows the fit of each profile.

In order to fit the observed SEDs, we used the SEDfitting code CIGALE (Noll et al. 2009). CIGALE creates synthetic spectra from the Bruzual \& Charlot (2003) stellar population models followed by the attenuation of the stellar 
Table 6. Mass models parameters for NGC 5257 and NGC 5258 from fits of the multi-wavelength rotation curve considering only points associated with circular motions.

\begin{tabular}{cccccccc}
\hline \hline Galaxy & $\begin{array}{c}\text { Type of } \\
\text { halo }\end{array}$ & $\begin{array}{c}\text { Maximal } \\
\text { disc }\end{array}$ & $\begin{array}{c}(M / L)_{\text {bulge }} \\
(I \text {-band })\end{array}$ & $\begin{array}{c}(M / L)_{\text {disc }} \\
(I-\text { band })\end{array}$ & $\begin{array}{c}R_{0} \\
(\mathrm{kpc})\end{array}$ & $\begin{array}{c}\rho_{0} \\
\left(10^{-3} M_{\odot} \mathrm{pc}^{-3}\right)\end{array}$ & $\chi^{2}$ \\
\hline \multirow{2}{*}{ NGC 5257 5} & pISO & Yes & 3.50 & 3.50 & 4.5 & 0.01 & 0.22 \\
& pISO & No & 1.75 & 0.50 & 2.6 & 0.40 & 0.12 \\
& NFW & Yes & 0.01 & 6.30 & 9.0 & 0.05 & 0.12 \\
& NFW & No & 0.50 & 0.50 & 16.8 & 0.04 & 0.19 \\
NGC 5258 & pISO & Yes & 3.20 & 3.10 & 5.8 & 0.06 & 0.45 \\
& pISO & No & 2.40 & 1.55 & 4.5 & 0.11 & 0.43 \\
& NFW & Yes & 4.30 & 3.70 & 45.0 & 0.71 & 0.70 \\
& NFW & No & 1.00 & 1.00 & 33.6 & 0.01 & 0.50 \\
\hline
\end{tabular}
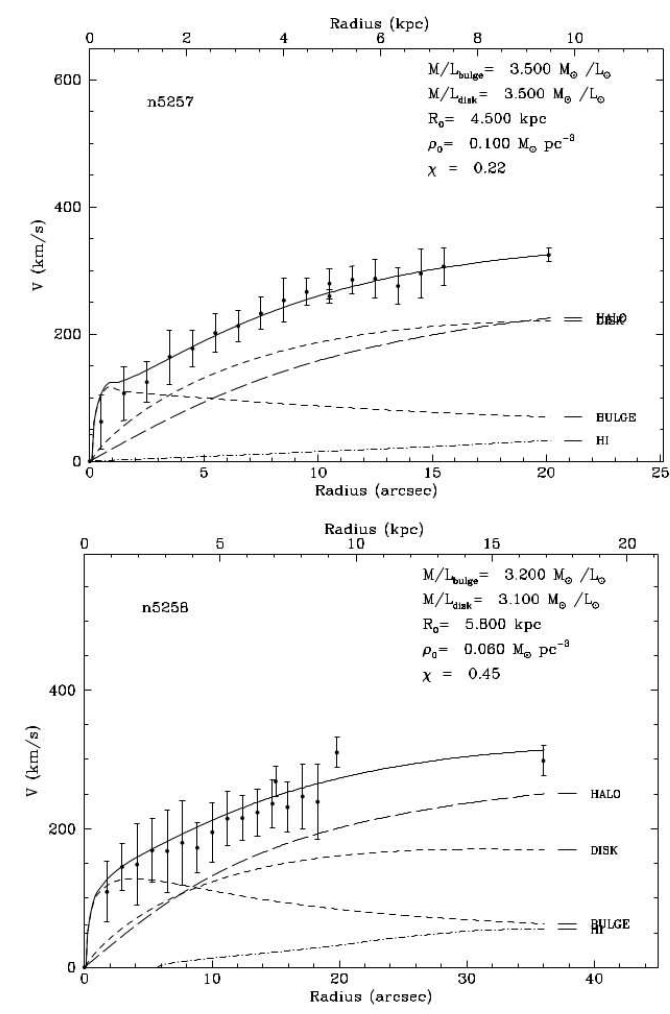

Fig. 12. Top panel: best mass model fit for the multi-wavelength rotation curve for NGC 5257. Bottom panel: best mass model fit for the multiwavelength rotation curve for NGC 5258.

population spectra using a synthetic Calzetti-based attenuation law (Calzetti et al. 2000) before adding the dust emission as given by the IR SED library built from the Dale \& Helou (2002) templates, which is particularly useful for fitting the SED of LIRGs. The code uses two single stellar populations with a Salpeter (1955) initial mass function (IMF) to reproduce an old and a young stellar population, since two stellar components have been found necessary to reconstruct more accurate SFRs in actively star-forming galaxies (e.g. Erb et al. 2006; Lee et al. 2009). The model assumes an SFH with exponentially decreasing SFR: $\tau_{1}$ and $\tau_{2}$ represent the $e$-folding time of the old and young stellar populations, respectively (see Giovannoli et al. (2011) for a detailed description). Initial parameters for the fit of the SED of each galaxy are shown in Table 7. The base consists of two single stellar populations (SSP) from Bruzual \& Charlot (2003) and a $\tau$-exponentially declining star formation history with $\tau=10.0$,
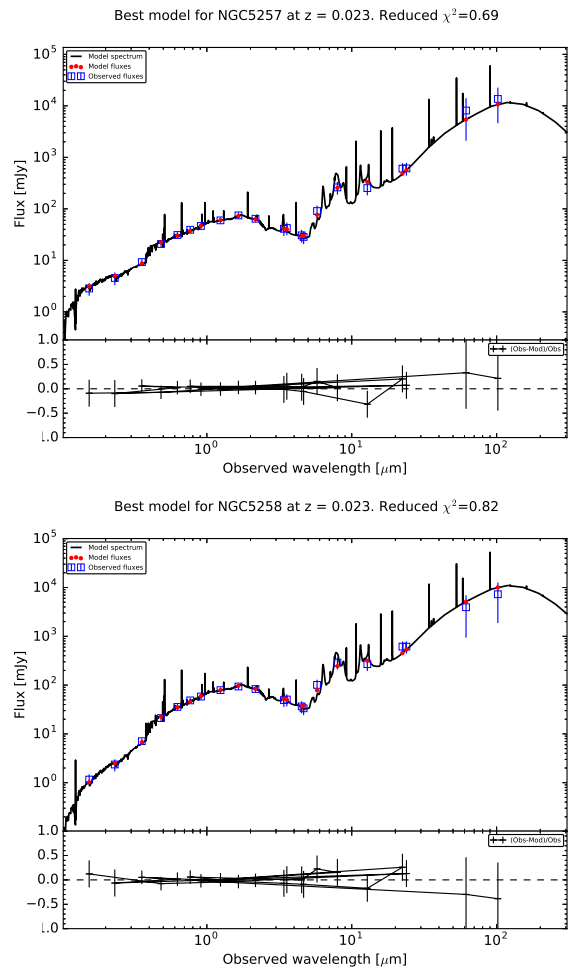

Fig. 13. Top panel: SED for NGC 5257. Bottom panel: SED for NGC 5258. A SED compiled using stellar synthesis model CIGALE (Noll et al. 2009) has been fitted to each SED. Empty blue squares correspond to observed fluxes, red dots indicate model fluxes, and the black solid line corresponds to the model spectrum. Bottom plots: normalized error fit versus wavelength for each galaxy.

20.0, 50.0, 100.0, 200.0, 400.0, 600.0, 800.0, 1000.0 Myr for the young population and $\tau=1,2,3,4,5,6,7 \mathrm{Gyr}$ for the old population. For both galaxies, an age of 13 Gyr was given as a fixed value for the old stellar population, while for the young stellar population, the initial age values were 10.0, 20.0, 50.0, 100.0, 200.0, 400.0, 600.0, 800.0, and 1000.0 Myr. Following Noll et al. (2009), a solar metallicity $Z=0.02 Z_{\odot}$ was selected. Initially the young versus old stellar population ratio was taken to be among the values $0.0001,0.001,0.01,0.1$, and 0.999 .

The reduced $\chi^{2}$ provided by the best fit model equals 0.69 and 0.82 for NGC 5257 and NGC 5258, respectively. The results for the best fit are shown in Table 8. For NGC 5257, the averaged SFR over $100 \mathrm{Myr}\left(42.3 \mathrm{M}_{\odot} \mathrm{yr}^{-1}\right)$ drops by almost a factor of two with respect to the averaged SFR over $10 \mathrm{Myr}\left(27.2 M_{\odot} \mathrm{yr}^{-1}\right)$, but the 
Table 7. SED fit. Input parameters of the CIGALE code.

\begin{tabular}{ll}
\hline \hline Parameter & \\
\hline$\tau$ of old stellar population models (Gyr) & $1,2,3,4,5,6,7$ \\
Ages of old stellar population models (Gyr) & 13 \\
$\tau$ of young stellar population models (Myr) & $5,10,20,50,100,200$ \\
Ages of young stellar population models (Myr) & $5,10,20,50,100,200$ \\
Fraction of young stellar population & $0.0001,0.001,0.01,0.1,0.999$ \\
Metallicity & 0.02 \\
IMF & Salpeter (1955) \\
Stellar population libraries & Bruzual \& Charlot (2003) \\
\hline
\end{tabular}

Table 8. SED fit. Final parameters

\begin{tabular}{lll}
\hline \hline Parameter & NGC 5257 & NGC 5258 \\
\hline$\tau$ of old stellar population $(\mathrm{Gyr})$ & 7 & 7 \\
Age of old stellar population* $(\mathrm{Gyr})$ & 13 & 13 \\
$\tau$ of young stellar population $(\mathrm{Myr})$ & 100 & 200 \\
Age of young stellar population $(\mathrm{Myr})$ & 200 & 400 \\
Fraction of young stellar population & 0.1 & 0.1 \\
Instantaneous SFR $\left(M_{\odot} \mathrm{yr}^{-1}\right)$ & 26.17 & 22.53 \\
Averaged SFR (over 10 Myr) $\left(M_{\odot} \mathrm{yr}^{-1}\right)$ & 27.23 & 22.93 \\
Averaged SFR (over 100 Myr) $\left(M_{\odot} \mathrm{yr}^{-1}\right)$ & 42.33 & 27.663 \\
Stellar mass of young population $\left(M_{\odot}\right)$ & $2.63 \times 10^{8}$ & $2.22 \times 10^{7}$ \\
Stellar mass of old population $\left(M_{\odot}\right)$ & $1.024 \times 10^{11}$ & $1.559 \times 10^{11}$ \\
Total stellar mass $\left(M_{\odot}\right)$ & $1.027 \times 10^{11}$ & $1.561 \times 10^{11}$ \\
Total gas mass $\left(M_{\odot}\right)$ & $4.18 \times 10^{10}$ & $6.43 \times 10^{10}$ \\
Metallicity & 0.02 & 0.02 \\
Reduced $\chi^{2}$ & 0.69 & 0.82 \\
\hline
\end{tabular}

Notes. ${ }^{(*)}$ Fixed values before running the code.

latter does not differ from the instantaneous SFR $\left(26.2 M_{\odot} \mathrm{yr}^{-1}\right)$. For NGC 5258, the variation between the averaged SFR over $100 \mathrm{Myr}\left(27.7 M_{\odot} \mathrm{yr}^{-1}\right)$ does not decrease significantly from the one computed over $10 \mathrm{Myr}\left(22.9 M_{\odot} \mathrm{yr}^{-1}\right)$ and instantaneously $\left(22.5 M_{\odot} \mathrm{yr}^{-1}\right)$. The stellar mass of the young population is more than ten times larger for NGC $5257\left(2.63 \times 10^{8} M_{\odot}\right)$ than for NGC $5258\left(2.22 \times 10^{7} M_{\odot}\right)$, while for the old population, masses are similar $\left(1.024 \times 10^{11} M_{\odot}\right.$ for NGC 5257 and $1.561 \times 10^{11} M_{\odot}$ for NGC 5258). Both total stellar mass and total gas mass are $50 \%$ larger for NGC $5258\left(M_{\star}=1.561 \times 10^{11} M_{\odot}\right.$ and $M_{\text {gas }}=$ $\left.6.43 \times 10^{10} M_{\odot}\right)$ than for NGC $5257\left(M_{\star}=1.027 \times 10^{11} M_{\odot}\right.$ and $\left.M_{\text {gas }}=4.18 \times 10^{10} M_{\odot}\right)$.

\section{Discussion}

The detailed study of the kinematics and dynamics of Arp 240 provides important information about the structure of each member of the pair, as well as about the stage of the encounter as a whole. The velocity fields of NGC 5257 and NGC 5258 show velocity distributions following those of rotating discs with kinematic disturbances in localized regions. For NGC 5257, non-circular velocities traced by large velocity dispersion values $\left(30 \mathrm{~km} \mathrm{~s}^{-1}\right.$ $55 \mathrm{~km} \mathrm{~s}^{-1}$ in top left panel in Fig. 5) are associated with starforming regions along the spiral arms, especially the large HII regions seen in the monochromatic image (bottom panel of Fig. 1). Large values are also seen on the tip of the eastern arm matching the beginning of the tidal tail. According to Epinat et al. (2010), the mean velocity dispersion of nearby isolated galaxies is $\sim 25 \mathrm{~km} \mathrm{~s}^{-1}$. In this case, the large values in NGC 5257 could be due to the induced ongoing SF processes, as well as the gravitational effects of the interaction with the companion. Regarding the residual velocities map of this galaxy, high values are detected near the minor axis of the galaxy close to the centre of the galaxy, $\theta \sim 90^{\circ}$ from the PA (bottom left panel of Fig. 5). In this region, low values of velocity dispersion are seen (top panel of Fig. 5). Considering this angle, the tangential component of the velocity in Eq. (3) in Fuentes-Carrera et al. (2004) can be neglected, indicating that an important fraction of non-circular velocities in this region lies in the radial direction on the plane of the galaxy, probably along the stellar bar. For NGC 5258, large velocity dispersion values of the ionized gas match the location of the large HII region on the western arm of the galaxy (top panel of Fig. 5). This is probably related to the intense star-forming processes in this region. Important residual velocity values are seen at the beginning of the tidal tail and the tidal bridge of this galaxy (bottom panel of Fig. 5), which along with the large velocity dispersion values could be tracing the effect of the gravitational perturbations by the companion on both spiral arms of the galaxy. Comparing the velocity dispersion and residual velocity fields for both galaxies, we notice an increase of velocity dispersion at the location of the HII regions, while an excess of residual velocities is observed at the tip of the arms. The different locations of important velocity dispersion values and residual velocity values is consistent with a scenario for which SF, shocks, and outflows increase closer to the disc and kinematical perturbations increase away from the disc in the spiral arms.

Globally, the RCs of both galaxies display a similar behaviour. The RCs remain symmetric up to a certain radius, then the 
rotation velocity on one side of the galaxy decreases, while for the other side, the velocity increases or remains constant. This type of bifurcation has been identified in simulations of major mergers (Pedrosa et al. 2008) where the time evolution of these asymmetries has been studied in a pair of galaxies during the first passage. According to those authors, the rotation curve asymmetries appear right at the pericentre of the first passage and occur within a small time interval. Therefore the presence of bifurcation in the $\mathrm{RC}$ of each galaxy in the pair could be used as an indicator of the pericentre occurrence and thus be related to the gravitational perturbation due to the presence of the equal-mass companion.

The pattern speed of NGC 5257 was derived using the TW method. This is an important probe for estimating the impact of gravitational perturbations at the resonance radii of a galaxy, whether these perturbations are due to the internal structure of the galaxy or to interaction with an almost equal-size companion as in the present case. Corsini et al. (2003) showed the TW method can be used to determine multiple pattern speeds. If more than one pattern is present, $\Omega_{p}$ will have contributions from the various patterns. In our case, the relatively large distance to the galaxies and the resulting spatial resolution of the velocity field did not allow us to do a detailed $\Omega_{\mathrm{p}}$ decomposition. We could, however, attempt to identify certain parts of the galaxy under the influence of certain density perturbation and derive the $\Omega_{p}$ considering only the average points falling in that region. For NGC 5257, $\Omega_{\mathrm{p}}$ matches the velocity value of the ionized gas where the RC bifurcates at $R_{\mathrm{bif}}=(17 \pm 2)^{\prime \prime}$. The fact that the velocity of the perturbation associated with the main disc of NGC 5257 matches the velocity of the ionized gas where the RC bifurcates indicates the extent of the effects of the gravitational perturbation by the massive companion galaxy on NGC 5257 versus the effects of the perturbations intrinsic to NGC 5257. Whatever density perturbation is present, its effect would be enhanced at this radius. We explored the possibility that $R_{\text {bif }}$, and thus $R_{\text {cor }}$, are related to the location of the SF processes in the galaxy by analysing the morphological and star-forming features at $R_{\text {bif }}$, that is at the position of the ellipse plotted on NGC 5257 in Figs. 1, 2, 7, and 6. The old stellar disc is confined by the ellipse, while the UV maxima in the emission lies outside the ellipse. The optical images indicate that the eastern spiral arm lies inside the ellipse. Outside the ellipse it appears to be tidally elongated to form the beginning of a tidal tail. Regarding the western arm of NGC 5257, the brightest region in most wavelengths lies just outside the ellipse.

This pair of galaxies is part of the sample studied by Smith et al. (2007) in which most of the interacting galaxies display nuclear and circumnuclear SF, implying that interactions can drive gas into the central region and trigger nuclear and circumnuclear SF before merging. However, in our case, both galaxies harbour all or most of the SF along their spiral arms. In particular for NGC 5257, the SF is confined to the spiral arms, close to the plotted ellipse. For NGC 5258, the SF processes are confined to the SW region of the disc along the southern spiral arm, while some gas seems to have been fueled to the central parts of the galaxy (seen in the 4.5, 8.0, and $60 \mu \mathrm{m}$ images).

Regarding the encounter, the apparent bridge between the two galaxies is seen in the $\mathrm{F} 814 \mathrm{~W}, 4.5$, and $8.0 \mu \mathrm{m}$ images. The bridge is broad and joins both galaxies, at least in projection. It is also seen in the HI image by Iono et al. (2005); however, it is not detected in any of the $\mathrm{H} \alpha$ images. The tidal tail in NGC 5257 is a broad feature seen in UV, optical, NIR, 4.5 , and $8.0 \mu \mathrm{m}$ images (Fig. 7). It is not very extended, even in the HI image by Iono et al. (2005). NGC 5257 does not look very perturbed, neither morphologically nor kinematically, by the presence of the massive companion, which could suggest this galaxy is suffering a retrograde encounter as shown by numerical simulations (Toomre \& Toomre 1972; Bournaud et al. 2004; D'Onghia et al. 2010). The bifurcation observed in its RC seems to be associated with the beginning of the tidal arms. On the contrary, the tidal tail of NGC 5258 is very broad and much more extended suggesting this galaxy is experiencing a prograde encounter. The relative orientation of the spin of the galaxies with the spin of the orbit could explain why NGC 5258 is more perturbed than NGC 5257. According to simulations by Holincheck et al. (2016), NGC 5257 is experiencing a retrograde encounter, and the pair has already experienced the first peripassage which occurred $\sim 250 \mathrm{Myr}$ ago. This time is in agreement with the findings by Pedrosa et al. (2008) where the bifurcation in the RC appears right after pericentre of the first passage and lasts $\sim 0.5 \mathrm{Gyrh}^{-1}$. Assuming the above orientation between both galaxies, the perturbation has been stronger for NGC 5258 and thus has experienced an induced SF episode before its companion, NGC 5257. Indeed the SF in NGC 5257 is still confined to the outer parts of the galaxy, particularly the spiral arms, while it is no longer the case for NGC 5258. This delay in the triggering of the SF could explain the difference in the $\tau$ values in the SED fit for each galaxy.

The stellar mass ratio derived from the SED fit indicates that NGC 5258 is more massive than NGC 5257. This mass ratio is derived for each galaxy as a whole, while the RC decomposition shows that at small radii $\left(0.37 R_{25}\right)$, NGC 5257 seems to be more massive than NGC 5258. This result is confirmed by the mass estimates using the Lequeux (1983) method. According to the simulations presented by Holincheck et al. (2016), Arp 240 is a 1:1 encounter, or a 1:2 encounter at most. Higher stellar mass at a large radius for NGC 5258 could imply that the SF on the outskirts of this galaxy has already happened and is currently fueling the centre of the galaxy, while NGC 5257 has a more massive young stellar population confined to the outer parts of the galaxy. This can be thus considered as an $\sim 1: 1$ encounter where the parameters of the encounter have determined a different SFH for each galaxy. Still both galaxies exhibit most SF far from their central parts.

For the DM halo fit for these galaxies, the outer points of both RCs are affected by the interaction in such a way that the DM halo fit is mainly associated with the inner parts of the galaxy. This corresponds to the part of the RCs before the bifurcation radii are observed. Though this reduces the amount of information that can be derived from the fit, previous works (Blais-Ouellette et al. 1999, 2001) have shown the importance of this part of the RC to infer the best DM halo model for individual spiral galaxies. In particular for Arp 240, it seems both RCs are best fit by a maximal disc and a pseudo-isothermal halo.

\section{Conclusions}

We have presented the kinematical and dynamical analysis of the interacting luminous infrared galaxy pair Arp 240 (NGC 5257/58). We used scanning Fabry-Perot interferometric $\mathrm{H} \alpha$ observations to study the motions of different gas components. Through a morpho-kinematical analysis using direct images in different wavelengths, we have traced the location of recent and ongoing SF in the context of the interaction. In the case of NGC 5257, the SF is spread over the whole disc of the galaxy, while for NGC 5258, the SF is mainly confined to a particular region in the southern arm where gas is being fueled towards the centre. Non-circular motions are detected in both galaxies. For 
both galaxies, in the inner parts of the discs, these motions are typically associated to spiral arms, and HII regions, and to streaming motion along the bar. For the outer parts of each galaxy, they are associated with the presence of the companion and the tidal response of the disc.

The pattern speed of NGC 5257 was computed using the TW method. The corotation radius, $R_{\text {cor }}$, matches the location in the galaxy where the RC bifurcates. This radius could be pinpointing regions in the galaxy that are reacting to a tidal gravitational perturbation. The SF seems closely related to gravitational perturbation of the disc. This hypothesis will be explored through dynamical models of interacting galaxies in which the effects of perturbations can be traced.

This is a case where significant SF is confined to the arms of the galaxies. This does not mean gas will not be more centrally concentrated in the future. However, our study shows that important SF can occur in the outer parts of interacting galaxies. Our programme for the study of interacting galaxy pairs includes future numerical simulations that will consider gas and will take into account the extended kinematical information derived from the scanning FP interferometry observations in order to map the SF processes in both galaxies and the evolution of the perturbation during the encounter.

Acknowledgements. We thank the staff of the Observatorio Astronómico Nacional (OAN-SPM) for their support during PUMA data acquisition and C. Carignan for letting us use his mass model. IFC thanks the financial support of IPN-SAPPI project 20181136 and CONACYT grant 133520. MR acknowledges financial support from grants DGAPA-PAPIIT (UNAM) IN103116 and CONACYT CY-253085. We acknowledge the use of the HyperLeda database (http://leda.univ-lyon1.fr) and the NASA/IPAC Extragalactic Database (NED). Some of the data presented in this paper were obtained from the Mikulski Archive for Space Telescopes (MAST). STScI is operated by the Association of Universities for Research in Astronomy, Inc., under NASA contract NAS5 26555. This research has made use of the NASA/IPAC Infrared Science Archive, which is operated by the Jet Propulsion Laboratory, California Institute of Technology, under contract with the National Aeronautics and Space Administration.

\section{References}

Amram, P., Marcelin, M., Boulesteix, J., \& Le Coarer, E. 1989, A\&AS, 81, 59 Arp, H. 1966, ApJS, 14, 1

Begeman, K. G. 1987, PhD Thesis, Kapteyn Institute, The Netherlands Bellocchi, E., Arribas, S., \& Colina, L. 2016, A\&A, 591, A85 Bergvall, N., Laurikainen, E., \& Aalto, S. 2003, A\&A, 405, 31 Blais-Ouellette, S., Amram, P., \& Carignan, C. 2001, AJ, 121, 1952 Blais-Ouellette, S., Carignan, C., Amram, P., \& Côté, S. 1999, AJ, 118, 2123 Bournaud, F., Combes, F., \& Jog, C. J. 2004, A\&A, 418, L27 Brassington, N. J., Zezas, A., Ashby, M. L. N., et al. 2015, ApJS, 218, 6 Brown, M. J. I., Moustakas, J., Smith, J.-D. T., et al. 2014, ApJS, 212, 18 Bruzual, G., \& Charlot, S. 2003, MNRAS, 344, 1000

Bushouse, H. A., Telesco, C. M., \& Werner, M. W. 1998, AJ, 115, 938

Calzetti, D., Armus, L., Bohlin, R. C., et al. 2000, ApJ, 533, 682

Casasola, V., Bettoni, D., \& Galletta, G. 2004, A\&A, 422, 941

Corsini, E. M., Debattista, V. P., \& Aguerri, J. A. L. 2003, ApJ, 599, L29

Dale, D. A., \& Helou, G. 2002, ApJ, 576, 159

Davies, L. J. M., Robotham, A. S. G., Driver, S. P., et al. 2015, MNRAS, 452, 616

Delgado-Serrano, R., Hammer, F., Yang, Y. B., et al. 2010, A\&A, 509, A78

de Vaucouleurs, G., de Vaucouleurs, A., Corwin, Jr., H. G., et al. 1991, Third Reference Catalogue of Bright Galaxies (New York, USA: Springer)

Dicaire, I., Carignan, C., Amram, P., et al. 2008, MNRAS, 385, 553

D’Onghia, E., Vogelsberger, M., Faucher-Giguere, C.-A., \& Hernquist, L. 2010, ApJ, 725, 353
Dopita, M. A., Pereira, M., Kewley, L. J., \& Capaccioli, M. 2002, ApJS, 143, 47

Ellison, S. L., Mendel, J. T., Scudder, J. M., Patton, D. R., \& Palmer, M. J. D. 2013, MNRAS, 430, 3128

Epinat, B., Amram, P., Balkowski, C., \& Marcelin, M. 2010, MNRAS, 401, 2113

Erb, D. K., Steidel, C. C., Shapley, A. E., et al. 2006, ApJ, 646, 107

Fathi, K., Toonen, S., Falcón-Barroso, J., et al. 2007, ApJ, 667, L137

Fathi, K., Beckman, J. E., Piñol-Ferrer, N., et al. 2009, ApJ, 704, 1657

Fuentes-Carrera, I., Rosado, M., Amram, P., et al. 2004, A\&A, 415, 451

Fuentes-Carrera, I., Flores, H., Yang, Y., et al. 2010, A\&A, 513, A43

Gabbasov, R. F., Repetto, P., \& Rosado, M. 2009, ApJ, 702, 392

Gabbasov, R. F., Rosado, M., \& Klapp, J. 2014, ApJ, 787, 39

Gao, Y., \& Solomon, P. M. 1999, ApJ, 512, L99

Georgakakis, A., Forbes, D. A., \& Norris, R. P. 2000, MNRAS, 318, 124

Giovannoli, E., Buat, V., Noll, S., Burgarella, D., \& Magnelli, B. 2011, A\&A, 525, A 150

Haas, M. R., Schaye, J., \& Jeeson-Daniel, A. 2012, MNRAS, 419, 2133

Hernandez, O., Wozniak, H., Carignan, C., et al. 2005, ApJ, 632, 253

Hernández-Toledo, H. M., \& Puerari, I. 2001, A\&A, 379, 54

Holincheck, A. J., Wallin, J. F., Borne, K., et al. 2016, MNRAS, 459, 720

Hudson, M. J., Gillis, B. R., Coupon, J., et al. 2015, MNRAS, 447, 298

Iono, D., Yun, M. S., \& Ho, P. T. P. 2005, ApJS, 158, 1

Ji, I., Peirani, S., \& Yi, S. K. 2014, A\&A, 566, A97

Kazantzidis, S., Mayer, L., Mastropietro, C., et al. 2004, ApJ, 608, 663

Knapen, J. H., Cisternas, M., \& Querejeta, M. 2015, MNRAS, 454, 1742

Krabbe, A. C., Rosa, D. A., Pastoriza, M. G., et al. 2017, MNRAS, 467, 27

Le Coarer, E., Rosado, M., Georgelin, Y., Viale, A., \& Goldes, G. 1993, A\&A, 280,365

Lee, S.-K., Idzi, R., Ferguson, H. C., et al. 2009, ApJS, 184, 100

Lequeux, J. 1983, A\&A, 125, 394

Liu, C. T., \& Kennicutt, Jr., R. C. 1995, ApJS, 100, 325

Martinez-Valpuesta, I., Aguerri, J. A. L., González-García, A. C., Dalla Vecchia, C., \& Stringer, M. 2017, MNRAS, 464, 1502

Merrifield, M. R., \& Kuijken, K. 1995, MNRAS, 274, 933

Mould, J. R., Huchra, J. P., Freedman, W. L., et al. 2000, ApJ, 529, 786

Navarro, J. F., Frenk, C. S., \& White, S. D. M. 1996, ApJ, 462, 563

Noll, S., Burgarella, D., Giovannoli, E., et al. 2009, A\&A, 507, 1793

Patton, D. R., Ellison, S. L., Simard, L., McConnachie, A. W., \& Mendel, J. T. 2011, MNRAS, 412, 591

Pedrosa, S., Tissera, P.B., Fuentes-Carrera, I., \& Mendes de Oliveira, C. 2008, A\&A, 484, 299

Planck Collaboration XIII. 2016, A\&A, 594, A13

Randriamampandry, T. H., Combes, F., Carignan, C., \& Deg, N. 2015, MNRAS, 454, 3743

Rodrigues, M., Hammer, F., Flores, H., Puech, M., \& Athanassoula, E. 2017, MNRAS, 465, 1157

Rosado, M., Langarica, R., Bernal, A., et al. 1995, Rev. Mex. Astron. Astrofis., 3,263

Sackett, P. D. 1997, ApJ, 483, 103

Salo, H., \& Laurikainen, E. 1993, ApJ, 410, 586

Salo, H., \& Laurikainen, E. 2000a, MNRAS, 319, 377

Salo, H., \& Laurikainen, E. 2000b, MNRAS, 319, 393

Salpeter, E. E. 1955, ApJ, 121, 161

Schreiber, C., Pannella, M., Elbaz, D., et al. 2015, A\&A, 575, A74

Scudder, J. M., Ellison, S. L., Momjian, E., et al. 2015, MNRAS, 449, 3719

Sersic, J. L. 1968, Atlas de Galaxias Australes (Córdoba, Argentina: Observatorio Astronómico)

Skrutskie, M. F., Cutri, R. M., Stiening, R., et al. 2006, AJ, 131, 1163

Smith, B. J., Struck, C., Hancock, M., et al. 2007, AJ, 133, 791

Sofue, Y., Wakamatsu, K.-I., Taniguchi, Y., \& Nakai, N. 1993, PASJ, 45, 43

Soifer, B. T., Boehmer, L., Neugebauer, G., \& Sanders, D. B. 1989, AJ, 98, 766

Springel, V., Wang, J., Vogelsberger, M., et al. 2008, MNRAS, 391, 1685

Toomre, A., \& Toomre, J. 1972, ApJ, 178, 623

Tremaine, S., \& Weinberg, M. D. 1984, ApJ, 282, L5

Warner, P. J., Wright, M. C. H., \& Baldwin, J. E. 1973, MNRAS, 163, 163

Wright, E. L., Eisenhardt, P. R. M., Mainzer, A. K., et al. 2010, AJ, 140, 1868

Yamashita, T., Komugi, S., Matsuhara, H., et al. 2017, ApJ, 844, 96

Yun, M. S., Reddy, N. A., \& Condon, J. J. 2001, ApJ, 554, 803

Zaragoza-Cardiel, J., Beckman, J. E., Font, J., et al. 2015, MNRAS, 451, 1307 